Published in final edited form as:

Semin Neurol. 2012 September ; 32(4): 374-400. doi:10.1055/s-0032-1331810.

\title{
Advanced Neuroimaging in Traumatic Brain Injury
}

\author{
Brian L. Edlow, M.D. ${ }^{1,2,3}$ and Ona Wu, Ph.D. ${ }^{3,4}$ \\ ${ }^{1}$ Department of Neurology, Massachusetts General Hospital, Boston, MA \\ ${ }^{2}$ Department of Neurology, Brigham and Women's Hospital, Boston, MA \\ ${ }^{3}$ Athinoula A. Martinos Center for Biomedical Imaging, Massachusetts General Hospital, \\ Charlestown, MA \\ ${ }^{4}$ Department of Radiology, Massachusetts General Hospital, Boston, MA
}

\begin{abstract}
Advances in structural and functional neuroimaging have occurred at a rapid pace over the past two decades. Novel techniques for measuring cerebral blood flow, metabolism, white matter connectivity, and neural network activation have great potential to improve the accuracy of diagnosis and prognosis for patients with traumatic brain injury (TBI), while also providing biomarkers to guide the development of new therapies. Several of these advanced imaging modalities are currently being implemented into clinical practice, whereas others require further development and validation. Ultimately, for advanced neuroimaging techniques to reach their full potential and improve clinical care for the many civilians and military personnel affected by TBI, it is critical for clinicians to understand the applications and methodological limitations of each technique. In this review, we examine recent advances in structural and functional neuroimaging and the potential applications of these techniques to the clinical care of patients with TBI. We also discuss pitfalls and confounders that should be considered when interpreting data from each technique. Finally, given the vast amounts of advanced imaging data that will soon be available to clinicians, we discuss strategies for optimizing data integration, visualization and interpretation.
\end{abstract}

\section{Keywords}

traumatic brain injury (TBI); susceptibility-weighted imaging (SWI); diffusion tensor imaging (DTI); high angular resolution diffusion imaging (HARDI); functional MRI (fMRI); traumatic axonal injury (TAI)

\begin{abstract}
Advances in neuroimaging over the past two decades have yielded novel insights about the structure and function of the human brain. These advanced imaging techniques have also shown great potential to improve clinical care for the estimated 1.7 million civilians who experience a traumatic brain injury (TBI) in the United States each year, ${ }^{1}$ as well as the hundreds of thousands of military personnel who have experienced a TBI since the start of the wars in Afghanistan and Iraq. ${ }^{2}$ To date, advanced neuroimaging in patients with TBI has largely focused on traumatic axonal injury (TAI), a specific subtype of TBI-related pathology. TAI is caused by shear-strain forces that occur as the brain rotates, accelerates, or decelerates within the skull at the moment of impact. ${ }^{3-5}$ Unlike other types of lesions within the broad spectrum of TBI pathology, which include skull fracture, epidural hemorrhage, subdural hemorrhage, subarachnoid hemorrhage, and contusions, TAI typically evades
\end{abstract}

Address for correspondence and reprint requests: Brian L. Edlow, M.D., Massachusetts General Hospital, Department of Neurology, 55 Fruit Street - Lunder 650, Boston, MA 02114 (bedlow@ partners.org). 
detection by computed tomography (CT) and conventional magnetic resonance imaging (MRI). ${ }^{6}$ As a result, the diagnosis of TAI is often made on an inferential basis when CT or conventional MRI findings are not commensurate with a patient's neurological deficits. Since TAI is not only the most difficult to detect type of lesion in TBI, but also the most common ${ }^{7}$ and neurologically devastating, ${ }^{8}$ significant attention has been directed toward the application of advanced neuroimaging techniques to the detection of TAI, a subject that will be the focus of this review.

Over the past several years, advanced neuroimaging techniques have dramatically improved the clinician's ability to detect TAI and have yielded new insights into its pathophysiology, as well as the mechanisms that enable recovery of neurological function after TAI. While the initial histopathological descriptions of TAI were limited to patients with severely altered consciousness, $,{ }^{9}, 10$ there is now a growing body of evidence from advanced imaging studies that TAI occurs across the entire spectrum of TBI severity, as discussed below. Moreover, recent histopathological investigations have confirmed that TAI may occur in patients with mild TBI. ${ }^{11,12}$ Based on these radiologic and pathologic findings, TAI is now believed to exist along a pathophysiologic spectrum that ranges in severity from transient disturbance of transmembrane ionic homeostasis, to reversible disruption of axoplasmic transport via neurofilament and microtubule networks, to primary or secondary axotomy. ${ }^{13,14}$ Accordingly, the clinical manifestations of TAI are believed to range from post-traumatic amnesia and inattention in patients with mild-to-moderate TBI to vegetative state or coma in patients with severe TBI. ${ }^{15}$ The reconceptualization of TAI as a pathophysiologic process that is not invariably "diffuse," but rather one that may be focal or multi-focal, has also led to important changes in nomenclature. Specifically, in the TBI neuroimaging guidelines recently proposed by the Federal Interagency Initiative on Common Data Elements for TBI, ${ }^{16,17}$ TAI is classified as "focal" ( $\leq 3$ foci of signal abnormality) or "diffuse" ( $>3$ foci). This classification system appropriately reflects the variable degree of TAI that may be observed in individual patients. ${ }^{16}$

As advances in neuroimaging have helped to redefine TAI and elucidate its complex pathophysiology, a major challenge that now confronts clinicians is how to apply these advanced imaging techniques to the benefit of their patients. With each new technology, there are a myriad of methodological factors that must be considered for accurate interpretation, and for some techniques the data acquisition and post-processing time may limit the feasibility of clinical implementation. This review aims to provide an overview of recent advances in structural and functional neuroimaging that are either immediately relevant to the clinical care of patients with TBI or are likely to be integrated into clinical practice in the near future. The methodological principles and clinical applications of each technique are described, with particular attention directed toward feasibility and implementation. For each advanced imaging technique, the pitfalls and limitations that may confound data interpretation are also discussed. Moreover, the specific recommendations of the Common Data Elements workgroup pertaining to each advanced imaging technique are presented, wherever applicable. ${ }^{16,17}$ Special considerations in advanced neuroimaging of military personnel with TBI, athletes with concussion, and patients with traumatic disorders of consciousness are also discussed. Finally, it should be emphasized that the distinction between "conventional" and "advanced" neuroimaging techniques is inherently arbitrary and evolving, given the rapid pace at which new technologies are being integrated into clinical practice. For the purpose of this review, we will consider a conventional imaging technique to be one that is widely available clinically, e.g. 2-dimensional non-contrast head CT, T2weighted MRI, T2-weighted fluid attenuated inversion recovery (T2-FLAIR) MRI, gradientrecalled echo MRI (GRE) and diffusion-weighted MRI (DWI) for which less than 6 diffusion-weighted directions are used. 


\section{Computed Tomography and Conventional Magnetic Resonance Imaging}

The use of CT and conventional MRI for diagnosis and prognostication in patients with TBI has been reviewed extensively ${ }^{6,18,19}$ and therefore will not be discussed in detail here, except to emphasize the subtypes of TBI lesions that are amenable to detection by CT and conventional MRI versus those that are not (i.e. TAI). CT continues to be the preferred technique for acute neuroimaging of patients with TBI because of its accessibility, speed of acquisition, and its ability to detect skull fractures and large intracranial hemorrhages that require urgent neurosurgical intervention (Fig. 1). CT grading systems such as the Marshall CT classification ${ }^{20}$ and Rotterdam CT score ${ }^{21}$ continue to be used in clinical practice, and elements of both grading systems have been incorporated into commonly used prognostic models, such as the International Mission for Prognosis and Analysis of Clinical Trials in TBI (IMPACT) ${ }^{22,23}$ and the Medical Research Council (MRC) CRASH $^{24}$ models. Recent studies suggest that sophisticated multivariate models using CT parameters may further improve outcome prediction. ${ }^{25,26} \mathrm{CT}$ is also an effective tool for identifying the subset of mild TBI patients (Glasgow Coma Scale [GCS] score of 13 to 15) who have non-operative lesions that warrant hospital admission, observation, and/or follow-up imaging. ${ }^{27}$

Conventional MRI may not be feasible during acute stage of TBI because of lack of access, prolonged data acquisition time, or clinical factors that preclude MRI (i.e. raised intracranial pressure or metallic implants). Nevertheless, conventional MRI utilizing T2-FLAIR, GRE, DWI and derived apparent diffusion coefficient (ADC) maps provides better detection of TAI than does CT (Fig. 2). ${ }^{28-31}$ Accordingly, conventional MRI has been shown to be a more useful prognostic tool than $\mathrm{CT} .{ }^{28,32,33}$ White matter ADC is particularly useful for predicting outcomes, with one recent study demonstrating that ADC values in the wholebrain white matter and in the corpus callosum correlate with functional outcome in severe TBI patients at the time of hospital discharge. ${ }^{34}$ Reduced ADC values in the corticospinal tracts and corpus callosum have also been observed in concussed athletes compared to controls. ${ }^{35}$ In addition, conventional MRI studies, particularly those utilizing DWI, ADC and GRE, have yielded important insights about TAI pathophysiology. For example, restricted diffusion of water, as indicated by DWI hyperintensity and ADC hypointensity, develops over a highly variable timeline in TAI, likely due to the variable pathophysiological changes that occur in the intracellular compartment depending on the severity of shear-strain forces. Unlike in ischemic stroke, in which the DWI and ADC signal changes associated with diffusion restriction appear within minutes and follow a stereotyped time course with pseudonormalization of the ADC signal by seven to ten days, ${ }^{36} \mathrm{TAI}-$ related diffusion restriction may occur in a delayed fashion and has been reported to persist up to 18 days post-TBI. ${ }^{37}$ Furthermore, whereas diffusion restriction is typically associated with irreversible, cytotoxic edema in ischemic stroke, there are rare reports of neurological recovery in TBI patients despite evidence of extensive TAI-related diffusion restriction. ${ }^{38}$ These observations are consistent with animal studies showing that TAI may be reversible when shear-strain forces do not cause acute, primary axotomy. ${ }^{14}$ Consequently, the terms "cytotoxic edema" and "neurotoxic edema" should be used with caution when describing TAI-related diffusion restriction, since these lesions are not invariably associated with secondary axotomy or neuronal death. Moreover, the dynamic nature and potential reversibility of TAI-related signal abnormalities on DWI/ADC suggest a longer window for therapeutic intervention as compared to ischemic stroke.

Just as the DWI/ADC sequence has helped to elucidate the complex and heterogeneous pathophysiology of non-hemorrhagic TAI, the GRE sequence has yielded important insights about hemorrhagic TAI. From a pathophysiological standpoint, it has long been recognized that the shear-strain forces that disrupt and/or sever axons in TAI also cause disruption of the brain's microvasculature, resulting in extravasation of blood (Fig. 3) ${ }^{39}$ Whereas CT 
studies in the 1990s found that microhemorrhages, or traumatic microbleeds (TMBs), are associated with approximately $20 \%$ of radiologically apparent TAI lesions, ${ }^{6}$ GRE studies subsequently demonstrated that up to $80 \%$ of TAI lesions may be associated with TMBs. ${ }^{31,40}$ While a TMB is not incontrovertible evidence of concurrent axonal injury, the biomechanical and pathophysiological links between vessel disruption and axonal disruption suggest that the presence of axonal injury can be reasonably inferred when a TMB is present in the same neuroanatomic region. Even if radiologic evidence of axonal pathology (i.e. signal change on T2 FLAIR or DWI) is not identified, the presence of a TMB is considered in clinical practice, research studies, and in the Common Data Element guidelines, as evidence of hemorrhagic TAI. ${ }^{16}$ Supporting this assumption are studies showing that the total number of TMBs correlates with admission GCS score, ${ }^{40-42}$ duration of post-traumatic unconsciousness, ${ }^{41,43}$ and degree of neurological recovery. ${ }^{41,43}$ Furthermore, individual TMBs have been shown to account for focal neurological deficits at 1 month post-TBI. ${ }^{43}$ Yet, while TMBs identified by GRE have been correlated with acute TBI severity and shortterm outcomes, prior studies have failed to consistently demonstrate GRE's utility as a tool for predicting long-term outcomes. ${ }^{31,40,43-45}$ These results highlight the need for advanced imaging techniques that are more sensitive for TMB detection and thus potentially more useful for prognosis.

\section{Advanced Structural Imaging Techniques}

\section{Susceptibility-weighted Imaging (SWI)}

The development of a 3-dimensional high-resolution susceptibility-weighted imaging (SWI) technique by Haacke and colleagues in $2004^{46,47}$ significantly enhanced the detection of TMBs in hemorrhagic TAI at clinically available field strengths of $1.5 \mathrm{~T}$. The increased sensitivity for TMB detection that SWI provides over standard 2-dimensional GRE images is due to advances in both data acquisition and data post-processing. Unlike the conventional GRE sequence, which relies solely upon T2*-weighted magnitude images to identify the susceptibility effects of extravasated blood, SWI combines both magnitude and phase data to produce an image with enhanced susceptibility contrast. SWI thereby increases the conspicuity between hemorrhage-related blood products and the surrounding brain parenchyma (Fig. 4). As a result of these methodological advances, SWI has been shown to detect more microbleeds than GRE does in patients with TAI. ${ }^{41,42,48}$ Furthermore, the total number and volume of TMBs detected by SWI correlate with functional outcomes after TBI, ${ }^{41}$ whereas earlier studies that used GRE to detect TMBs failed to consistently demonstrate such a correlation. ${ }^{31,40,44,45}$ The reason(s) for SWI's increased predictive value have yet to be fully elucidated. It remains unclear whether it is the overall number of TMBs or the presence of TMBs in particular neuroanatomic locations that are most predictive of outcome. Tong and colleagues demonstrated that SWI is especially sensitive at detecting TMBs in the brainstem, ${ }^{41,48}$ a region in which unilateral and bilateral lesions are associated with odds ratios of 8 and 182, respectively, for poor outcome on the Glasgow Outcome Scale-Extended (GOSE). ${ }^{32}$ It is therefore possible that SWI improves outcome prediction by identifying TMBs in neuroanatomic regions where lesions have a particularly high prognostic significance.

In interpreting SWI data in patients with TBI, there are several important methodological factors that should be considered. The first and most important is the magnetic field strength at which the SWI data are acquired. Just as cerebral microbleeds (CMBs) caused by hypertension or cerebral amyloid angiopathy are more readily detected at higher field strength, ${ }^{49,50}$ TMBs are easier to detect at 3 Tesla as compared to $1.5 \mathrm{Tesla}^{51}$ due to increased sensitivity to susceptibility effects at higher field strengths. However, the utility of SWI over GRE at higher field strengths such as $3 \mathrm{~T}$ or $7 \mathrm{~T}$ is still debated. ${ }^{52}$ Some argue that the additional time required for a 3-dimensional acquisition may not be necessary, and 
longer acquisition times lead to greater likelihood of artifacts due to patient motion. Second, the in-plane spatial resolution and the distance between adjacent slices (i.e. interslice gap) may significantly affect the number of TMBs that are detected, since higher spatial resolution decreases volume averaging and thereby increases TMB detection. ${ }^{53}$ Thus, SWI data obtained from different patients or longitudinally in a single patient can only be reliably compared when magnetic field strength and imaging acquisition parameters are held constant.

Another important consideration when interpreting SWI results in patients with TBI is that consensus-based criteria for defining a TMB have yet to be established. Unlike in studies of hypertensive or amyloid-related microbleeds, for which standardized CMB criteria have been proposed, ${ }^{49}$ investigations of TBI patients have applied variable size criteria for defining TMBs. Sheid and colleagues defined TMBs as ranging from 1 to $15 \mathrm{~mm}$ in diameter, ${ }^{40,44,51}$ while other investigators have used diameter thresholds of $>2 \mathrm{~mm},{ }^{43}<10$ $\mathrm{mm},{ }^{42}$ or no size threshold at all. ${ }^{31,45}$ Notably, the Common Data Elements guidelines do not specify TMB size criteria. ${ }^{16}$ Until greater standardization of clinical protocols and more widespread implementation of SWI occurs, it may be prudent to refrain from proposing TMB size criteria, since comparative studies using GRE and SWI have shown that the two techniques provide different measurements of TMBs. Specifically, TMBs tend to be larger on GRE than on SWI due to greater "blooming" artifact associated with the former. ${ }^{48}$

Further complicating the effort to reach a consensus regarding the definition of a TMB is the possibility that hemorrhagic axonal injury lesions may be larger than $1 \mathrm{~cm}$, which calls into question the term "micro" in TMB. Moreover, since "gliding contusions" that occur at the grey-white matter junction are believed to be caused by the same shear-strain forces that cause TMBs, the distinction between TMBs and gliding contusions may be an arbitrary one that unnecessarily splits two pathophysiologically related entities into separate radiologic categories. Finally, while CMB grading scales such as the Microbleed Anatomical Rating Scale (MARS) $)^{54}$ and Brain Observer MicroBleed Scale (BOMBS $)^{55}$ have been validated by inter-rater reliability analyses, no such scale has been validated for TMBs. Thus, while SWI has already been implemented into routine clinical practice in many centers and is currently listed as a Tier 1 sequence in the Common Data Element guidelines, additional studies are needed to develop standardized TMB grading systems.

\section{Diffusion Tensor Imaging (DTI)}

Diffusion tensor imaging (DTI) is a natural extension of DWI. ${ }^{56}$ The development of DTI by Pierpaoli and Basser in the late 1990s revolutionized neuroimaging of white matter in the human brain and led to significant new insights about TAI pathophysiology in vivo. ${ }^{57,58}$ The fundamental principle upon which DTI is based is that the self-diffusion (i.e Brownian motion) of water molecules in the brain is directionally dependent. Water molecules within and around myelinated axons tend to diffuse in a directional (anisotropic) manner along the axis of the axon, whereas water molecules in grey matter or CSF tend to diffuse in a less directional (isotropic) manner. Assuming that the diffusion of water molecules in the brain is directionally dependent, it can be characterized by a tensor with six degrees of freedom. ${ }^{59}$ Whereas DWI attempts to measure mean diffusivity or apparent diffusion coefficient, DTI characterizes the shape (i.e. directionality) of the diffusion tensor. From a methodological standpoint, measuring the diffusion tensor requires the application of directional diffusion gradients in at least six non-collinear directions, as well as the acquisition of at least one non-diffusion-weighted (i.e. $\mathrm{b}=0 \mathrm{sec} / \mathrm{mm}^{2}$ ) measurement. From the diffusion tensor, several scalar quantitative metrics of water diffusion can be calculated. ${ }^{60}$ One of the most commonly used is fractional anisotropy (FA). In this unitless scale ranging from 0 to 1 , completely non-directional or isotropic diffusion is defined as FA=0, and completely directional or anisotropic diffusion is defined as $F A=1$. Figure 5 provides examples of a 
greyscale FA map, a color-coded FA map, and a tensor map. Figure 6 demonstrates how TAI may alter a color FA map and how these alterations provide insights into the structural basis for neurological deficits.

The feature of DTI-derived scalar metrics that is of particular relevance to TBI is their ability to detect structural changes in the white matter axons that are the pathophysiologic substrate of TAI. Low FA in the white matter has been correlated with histopathological evidence of TAI in experimental animal models. ${ }^{61,62}$ In human patients with TBI, FA values have been shown to be lower than in healthy control subjects in white matter regions susceptible to TAI, including the corpus callosum, ${ }^{45,63-73}$ internal capsule, ${ }^{64,65,67-69}$ centrum semiovale, ${ }^{64,69}$ anterior corona radiata, ${ }^{45,74}$ uncinate fasciculus, ${ }^{72-75}$ inferior longitudinal fasciculus, ${ }^{45,68,72,73}$ cingulum bundle, ${ }^{45,73,76}$ fornix, ${ }^{68,72,73}$ and brainstem. ${ }^{77,78}$ Low FA in these white matter regions has also been associated with a broad range of neurological deficits in patients with mild, ${ }^{45,67,71,72,74,75,79-82}$ moderate $65,66,72,82,83$ and severe TBI. ${ }^{65,68,73,78,82,84,85}$ Moreover, FA in specific white matter bundles correlates with neurocognitive functions that are known to be associated with those bundles. For example, memory has been correlated with FA in the uncinate fasciculus, superior longitudinal fasciculus, and fornix, ${ }^{73,74}$ attention with FA in the anterior corona radiata, ${ }^{74}$ and executive function with FA in the dorsolateral prefrontal region. ${ }^{80}$ DTI may also provide evidence of neural plasticity during recovery. For example, Sidaros and colleagues demonstrated that longitudinal increases in white matter FA correlated with recovery of neurological function in patients with severe TBI undergoing rehabilitation. ${ }^{84}$ Perhaps most strikingly, Voss and colleagues described a rare case of late recovery from the post-traumatic minimally conscious state (19 years post-injury), in which a longitudinal FA increase in the cerebellar vermis was associated with improvements in axial motor function as well as a longitudinal increase in resting metabolism in the vermis on ${ }^{18} \mathrm{~F}$-fluorodeoxy-glucose (FDG)-positron emission tomography (PET). ${ }^{86}$

The growing body of evidence that FA provides a biologically valid and functionally relevant assessment of white matter integrity suggests that clinical implementation of DTI is rapidly approaching. Indeed, the DTI sequence is currently listed in Tier 2 of recommended protocols in the Common Data Elements guidelines. ${ }^{16}$ Furthermore, the United States military has delivered MRI scanners to Afghanistan to detect TAI in military personnel exposed to blast-related or blunt head trauma. ${ }^{87}$ Yet, as the clinical community moves toward integration of DTI into routine practice in coming years, there are several methodological factors that should be considered. These factors can broadly be categorized as pertaining to data acquisition or data post-processing.

From a data acquisition standpoint, an important methodological issue is that of reproducibility of the quantitative anisotropy and diffusivity values. Both hardware (i.e. the MRI scanner) and software (i.e. the pulse sequence) may affect the characterization of the diffusion tensor. With regard to hardware, the degree to which a particular type of MRI scanner alters quantitative diffusion measurements, even using the same magnetic field strength and the same DTI sequence, has yet to be determined. Potential effects of the scanner on the diffusion measurements include inconsistent shimming, gradient miscalibration, and gradient non-linearity, each of which may lead to signal attenuation and/ or inconsistent measurements. ${ }^{60}$ These concerns about scanner-related effects on diffusion measurements were recently articulated in a letter to the editor of the New England Journal of Medicine in response to Mac Donald and colleagues' landmark study using DTI to identify blast-related white matter injury in United States military personnel (see TBI in Military Personnel section below for additional discussion). ${ }^{76}$ In this study, the blastexposed TBI cohort and control cohort were scanned initially in Landstuhl, Germany and then subsequently in St. Louis, United States. Both scans were performed on 1.5 Tesla 
Siemens Magnetom Avanto MRI scanners. Yet, in their letter, Drs. Xydakis, Butman, and Pierpaoli point out that the differences in cingulum bundle anisotropy between the MRI scanners (approximately 0.06 for each cohort) exceeded the differences between the cohorts on each scanner (approximately 0.02 at each time point). ${ }^{88}$ As a result of this concern for scanner-related differences in DTI measurements of anisotropy, some investigators have argued that anisotropy measurements from TBI patients should be normalized to anisotropy measurements acquired on controls using the same MRI scanner. Clearly, the need to normalize data on each scanner would represent a major barrier to widespread clinical utilization of DTI scalar metrics, and therefore, the effect of scanner type and magnetic field strength on DTI quantitative measurements is currently an active area of research. Even if standardized hardware and software are ultimately used in DTI analyses, white matter anisotropy may still vary with gender ${ }^{89}$ and age, ${ }^{90}$ and therefore normalization according to these demographic characteristics may be necessary. Thus, the utility of DTI scalar metrics in prognosticating outcomes on an individual patient basis is still up for debate. Of the various scalar metrics, FA currently appears most useful for diagnosis, prognosis, and exploring mechanisms of brain injury and recovery.

Another important consideration in the data acquisition stage of DTI is the number of directional diffusion gradients that are used to measure FA. Although DTI data can be acquired with as few as 6 diffusion-encoding directions, Jones demonstrated that at least 20 directional measurements may be needed to generate accurate, reproducible measurements of FA. ${ }^{91}$ Adding further complexity to the question "what is the right DTI sequence to use clinically" are the results of recent studies showing that even more diffusion directions (i.e. $>30$ ) may be needed to produce reliable diffusion tensor tractography results (see Diffusion Tensor Tractography section below). ${ }^{91-93}$ At the present time, the Common Data Element guidelines recommend 30 diffusion directions for DTI scans performed on 1.5 Tesla MRI scanners. Alternatively, either 64 diffusion directions or 12 diffusion directions with 5 averages are recommended for DTI scans on 3 Tesla MRI scanners. ${ }^{16}$ Regardless of which DTI sequences are ultimately incorporated into clinical MRI protocols, it is essential that rigorous assessment of DTI data quality is performed prior to the interpretation of FA measurements. This data quality assessment should include a careful review of the preprocessed diffusion maps. Patient motion during a DTI scan can significantly alter FA measurements, which argues for minimizing the time of data acquisition whenever possible. Reduction of DTI data acquisition time is currently an active area of investigation ${ }^{94,95}$ that will be important to facilitating clinical implementation. Other data acquisition considerations are the degree of diffusion-weighting that is used (i.e. $b$-value), the spatial resolution (i.e. voxel size), and the use of monopolar or bipolar sequences to correct for eddy current distortions. ${ }^{96}$

Methodological considerations during the statistical analysis stage of evaluating DTI metrics have an equally substantial impact on the validity of the data. The mean FA within a white matter bundle can be measured using a variety of methods, including manual tracing of a region of interest (ROI), automated segmentation of an ROI, and voxel-based analysis. Manual ROI placement has been used frequently in DTI studies of patients with $\mathrm{TBI}^{45,74,76,84,97}$ and in patients with severe TBI this approach may be more feasible than template-based approaches, because acute tissue shifts and chronic atrophy may preclude automatic segmentation of white matter tracts. However, manual tracing of white matter ROIs can be time consuming and requires neuroanatomic expertise, which limits the feasibility of this approach in clinical practice. Both manual and automated segmentation of white matter ROIs are susceptible to a variety of errors, including volume-averaging of FA in voxels that contain both white matter and nearby non-white matter structures. Voxelbased analysis may be performed more rapidly and provides the benefit of increased 
sampling (i.e. whole-brain voxel-based analysis), but this technique is susceptible to false positive findings and therefore requires correction for multiple comparisons. ${ }^{67,98}$

An elegant method for evaluating DTI metrics that addresses many of the aforementioned limitations was proposed by Smith and colleagues in 2006 with the introduction of tractbased spatial statistics (TBSS). In this voxel-based technique, FA is measured not on all voxels within the brain, but rather on voxels within a standardized white matter skeleton. ${ }^{99,100}$ Because the white matter skeleton only contains the central core of each white matter tract, volume-averaging errors are reduced. TBSS has been used to detect white matter injury in patients with mild, ${ }^{98,101-103}$ moderate, ${ }^{85,102}$ and severe TBI. ${ }^{73,85,102,104,105}$ TBSS analyses of white matter FA have also been shown to be consistent with FA results from whole-brain voxel-based DTI analyses. ${ }^{85}$ Given its methodological advantages, TBSS is becoming more commonly utilized in DTI studies of patients with TBI. However, since TBSS relies upon registration of each individual patient's DTI data to a template, the utility of the technique in the presence of large brain deformations, as often occurs in the case of severe TBI, has yet to be demonstrated.

With regard to data processing platforms, a variety of tools exist for analyzing DTI data, including the following software packages that are available to the academic community without charge: FMRIB Software Library (FSL), ${ }^{106,107}$ Diffusion Toolkit and Trackvis, ${ }^{108}$ DTI Studio, ${ }^{109}$ and 3D Slicer. ${ }^{110}$ While a discussion of the unique analysis pipelines in each program is beyond the scope of this review, we wish to underscore the point that the analytic methods employed by these programs may differ. Therefore, comparisons of data analyzed with different programs should be performed with caution. Finally, if DTI data are to be integrated into clinical practice, it remains to be determined whether the data should be analyzed in each TBI patient's "native space," or whether it will be more useful to normalize each patient's DTI data to a standard space, such as the coordinate systems of the Talairach and Tournoux atlas, ${ }^{111}$ or the more commonly used Montreal Neurological Institute atlas (MNI152 also known as ICBM152) ${ }^{112}$ that is distributed directly from the MNI ${ }^{113}$ or ICBM $^{114}$ and with many software packages such as FSL, ${ }^{115}$ Statistical Parametric Mapping (SPM), ${ }^{116}$ and MRIcron. ${ }^{117}$ Normalization enables population-based analyses and group comparisons of DTI data and is typically feasible in patients with mild-to-moderate TBI. In contrast, it can be difficult to accurately coregister DTI data in severe TBI patients with tissue shifts and mass effects. State-of-the-art coregistration techniques are currently being developed to address this issue. ${ }^{118-120}$

\section{Diffusion Tensor Tractography}

The development of diffusion tensor tractography by Mori, ${ }^{121}$ Jones, ${ }^{122}$ Basser, ${ }^{123}$ and others in the late 1990s provided the opportunity for in vivo 3-dimensional analysis of white matter connectivity in the human brain. The basic principle upon which tractography is based is that as long as the primary diffusion directions (primary eigenvectors) of the tensors in adjacent voxels are directionally coherent, these tensors can be considered as being part of a single fiber tract. A fiber tract can therefore be conceptualized as a "streamline" of connected vectors along a single deterministic path, hence the term "deterministic streamline tractography." Fiber tracts are typically calculated by manually tracing or automatically segmenting a white matter ROI, such as the corpus callosum, and then using this ROI as a "seed" for the generation of 3-dimensional fiber tracts passing through it (Fig. 5D). To virtually dissect two different bundles of fiber tracts that pass through the same region of white matter, a two-ROI approach may be used, as described by Catani and de Schotten. ${ }^{124}$ For example, the uncinate fasciculus fiber tracts cannot be isolated by tracing a single ROI in the external capsule, because the inferior fronto-occipital fasciculus also courses through this region. Thus, to virtually dissect the uncinate fasciculus from the inferior frontooccipital fasciculus, one can trace ROIs in the anterior temporal lobe and the external/ 
extreme capsules and then perform a tractography algorithm in which only the fiber tracts that pass through both ROIs are displayed.

Regardless of which ROI methodology or tractography algorithm is being used, it is important to emphasize that tractography is an inferential technique in which white matter tracts are reconstructed on the basis of water diffusion measurements. The number of axons that corresponds to a single fiber tract remains unknown. Furthermore, the number of fiber tracts that is calculated in any tractography analysis is significantly affected by data acquisition parameters, particularly the spatial resolution (i.e. voxel size). Although studies have begun to validate tractography with "gold-standard" histopathology results in human spinal cord samples obtained at ultra-high resolution, ${ }^{125}$ all tractography results should be interpreted with caution given the inherent limitations of the technique, as discussed below.

The application of diffusion tensor tractography to the study of TAI is based on the ability of tractography to identify alterations in white matter connectivity, as well as changes in the mean FA, number of fiber tracts, average tract length, and total volume of a white matter bundle. Wang and colleagues found that early diffusion tensor tractography (mean day 7) identified fiber tract damage in the corpus callosum, fornix, and cerebral peduncle projections, as indicated by lower mean FA, fiber volume, and/or fiber number, in 12 patients with severe TBI as compared to age- and gender-matched controls. ${ }^{126}$ Furthermore, mean FA, fiber number and fiber length in subregions of the corpus callosum correlated with TBI patients' functional outcome scores on the GOSE at a mean follow-up of 8 months. A subsequent longitudinal study by the same laboratory involving 28 patients with mild-tosevere TBI demonstrated that diffusion tensor tractography identifies changes in structural connectivity between the acute (day 0-9) and chronic (6-14 month) periods, and that DTI measurements of structural connectivity in both the acute and chronic periods predicted patient performance on a variety of neurocognitive tests. ${ }^{127}$ In another diffusion tensor tractography study performed at 8 months post-TBI, grey matter atrophy correlated with the presence of damage in associated white matter tracts (i.e. hippocampal volume correlated with mean FA in the fornix). ${ }^{128}$ These correlations between the spatial extent of grey matter injury and the degree of damage to associated white matter bundles are consistent with recent histopathological analyses linking cortical atrophy to TAI-related damage in underlying white matter. ${ }^{129}$

Newcombe and colleagues provided additional support for the utility of diffusion tensor tractography as a diagnostic and prognostic tool in TBI by demonstrating that patients in a post-traumatic vegetative state (VS) have a different neuroanatomic distribution of white matter injury than patients in a post-anoxic VS. ${ }^{77}$ Specifically, the preferential damage of brainstem fiber tracts revealed by diffusion tensor tractography in the post-traumatic VS patients is consistent with histopathological and biomechanical studies demonstrating that the brainstem is susceptible to rotational shear-strain forces in severe TBI. $3,8,130,131$ Similarly, diffusion tensor tractography studies of moderate and severe TBI patients demonstrate that the corpus callosum, which is also known to be susceptible to shear-strain forces in TAI, ${ }^{8}$ undergoes volume loss, shortening of fiber tracts, a decrease in mean FA and a decline in total tract number in the subacute and chronic stages of injury. ${ }^{63,68,72}$ Abnormalities in mean FA within callosal fiber tracts have also been demonstrated in patients with mild TBI during the acute ${ }^{132}$ and subacute ${ }^{72}$ stages of injury, suggesting that a variety of quantitative metrics provided by diffusion tensor tractography yield important information about axonal integrity across the clinical spectrum of TBI severity.

Yet, despite preliminary evidence that diffusion tensor tractography may be used to detect TAI and predict outcomes in patients with TBI, major obstacles and challenges to clinical implementation remain. The results of any tractography analysis depend significantly upon 
the data acquisition and post-processing parameters, and therefore tractography results must always be interpreted in the context of the analytic techniques that are being utilized. Several of these methodological factors have been discussed above in the section on DTI, but there are additional considerations that are unique to diffusion tensor tractography. First, with regard to data acquisition, it is important in any tractography analysis to consider the $b$ value (units $=\mathrm{sec} / \mathrm{mm}^{2}$ ), which reflects the strength of the directional diffusion gradients and the duration over which these gradients are applied. High $b$ values (i.e. $>2000 \mathrm{sec} / \mathrm{mm}^{2}$ ) have been shown to preferentially affect ADC values and not FA measurements in human white matter. ${ }^{133-135}$ However, the $b$ value strongly influences the angular resolution of any tractography analysis. Thus, the $b$ value will affect the overall detection rate of fiber tracts, and hence all quantitative metrics that are derived from a tractography analysis (i.e. mean tract FA, number of fiber tracts, tract length, etc.). Second, smaller voxel size (i.e. greater spatial resolution) allows for more accurate identification of crossing or branching fibers that travel in close proximity to one another. Third, when interpreting structural connectivity data from a diffusion tensor tractography study, it is important to remember that the number and neuroanatomic location of fiber tract disruptions reflects termination criteria that are prespecified during the post-processing stage and are therefore not necessarily indicative of TAI. One may specify, for example, that a fiber tract will terminate when it reaches a voxel with a high angle of curvature (typically $>40$ to 60 degrees) or a voxel with low fractional anisotropy (typically $<0.15$ to 0.25 ). If extracellular edema has caused the FA to decline below the FA threshold in particular voxel, a fiber tract will terminate in that voxel even in the absence of any TAI. This well recognized phenomena of extracellular edema confounding diffusion tensor tractography connectivity analyses has led to the development of novel post-processing techniques aimed at eliminating the contribution of extracellular water content from each voxel. ${ }^{136}$ Nevertheless, until free-water extraction methods are further validated and integrated in DTI analyses, one must interpret DTI tractography results with caution when there is evidence of extracellular edema on the T2 FLAIR and/or ADC maps.

The potential confounding effect of extracellular edema on DTI measurements of FA and diffusion tensor tractography measurements of white matter connectivity can more broadly be considered in the context of current debates about the optimal timing of data acquisition. If DTI scalar metrics and diffusion tensor tractography are to be integrated into clinical practice, clinicians will need to consider how the dynamic pathophysiological changes associated with TAI will affect data interpretation. In the acute stage of TAI, white matter FA changes are variable, with studies showing both increases ${ }^{132,137}$ and decreases ${ }^{65,126}$ in FA. These heterogeneous FA changes in the acute stage of TAI may be attributable to the differential effects of intracellular and extracellular edema on FA. In the former, FA may increase, since more water molecules are trapped in the intracellular compartment where diffusion preferentially occurs along the axis of the axon. In the latter, FA may decrease, since more water molecules are located in the extracellular compartment, where diffusion is more isotropic (non-directional). Given that complete axonal transection and incomplete, non-disruptive axonal injury do not have clearly distinguishable profiles of intracellular and extracellular edema, the heterogeneous FA responses observed in the acute stage of TAI can make outcome prediction difficult. In other words, since the pathophysiologic and radiologic profiles of complete (irreversible) and incomplete (reversible) TAI in the human brain are incompletely understood, the fate of any region of white matter affected by TAI is difficult to determine in the acute period. Moreover, individual patients may contain multiple TAI lesions with variable increases and decreases in FA, further complicating the assessment of individual lesions.

In contrast to the variable effects of acute TAI on FA, subacute and chronic TAI appears to have a more predictable effect on FA, with studies consistently showing that a decline in FA 
correlates with poor neurocognitive test performance or poor GOSE scores. ${ }^{45,74,78,82,84,85,97}$ For this reason, it is possible that DTI and diffusion tensor tractography will provide more clinically relevant and readily interpretable data in the subacute period than in the acute period. Ultimately, clinicians will need to balance the competing goals of obtaining advanced imaging data early enough to guide diagnosis, prognosis, and therapeutic decisionmaking and acquiring the data late enough that the confounding effects of acute edema are minimized.

\section{High Angular Resolution Diffusion Imaging (HARDI) Tractography}

Techniques that model multiple fiber orientations within a single voxel have a major methodological advantage over diffusion tensor tractography in their ability to resolve crossing fibers. In diffusion tensor tractography, a single tensor is used to represent the primary diffusion direction within each voxel. This single tensor model of water diffusion does not sufficiently characterize tissue microstructure in brain regions with crossing fibers, which are abundant in the human brain. ${ }^{138}$ In regions of complex fiber architecture, the multiple, distinct water diffusion vectors are averaged into a single tensor that does not accurately represent the multi-directional nature of the underlying tissue structure. To solve this problem of crossing fibers and more accurately measure multi-directional water diffusion within each voxel, several sophisticated diffusion acquisition and analysis techniques have been proposed. These techniques include diffusion spectrum imaging (DSI) ${ }^{139}$ Q-ball imaging (QBI), ${ }^{140,141}$ multi-tensor MRI, ${ }^{142}$ persistent angular structure MRI, ${ }^{143}$ spherical deconvolution, ${ }^{144}$ and composite hindered and restricted model of diffusion (CHARMED) MRI. ${ }^{145}$ From each of these types of diffusion datasets one can calculate an orientation distribution function (ODF), which is a probabilistic representation of multidirectional water diffusion within a voxel. The ODF thereby enables the modeling of multiple bundles of fiber tracts that cross within a voxel. Of note, ODFs have been shown to correspond with "gold standard" histopathological results in rodent brain tissue, ${ }^{146}$ and DSI tractography results have been validated with autoradiographic labeling of white matter tracts in non-human primates. ${ }^{147}$ A detailed discussion of each of these sophisticated techniques is beyond the scope of this review and can be found elsewhere. ${ }^{148,149}$ Although there is not currently a consensus on the terminology used to describe this broad group of sophisticated diffusion analysis techniques, we use here the general term high angular resolution diffusion imaging (HARDI), consistent with the nomenclature in Hess and Mukherjee's review on this subject, ${ }^{150}$ because each technique aims to optimize the angular resolution of the intra-voxel water diffusion measurements. Universally accepted standards for HARDI data acquisition have not been established to date.

The application of HARDI tractography to the study of TBI is in its infancy but has already shown great promise to advance understanding about TBI pathophysiology and mechanisms of recovery (Fig. 7). Shin and colleagues recently used DSI to study the connectivity of premotor and primary motor pathways in a severe TBI patient whose functional motor deficits corresponded with the precise localization of fiber tract disruptions. ${ }^{151}$ These connectivity data were reproducible on repeated measurements and were far more specific in identifying the injured fiber pathways than were DTI measurements of FA performed in the same patient. This case analysis also highlighted an important advantage of DSI and other HARDI techniques over diffusion tensor tractography, which is a reduction in the number of neuroanatomically inaccurate fiber pathways that are generated due to poor resolution of crossing fibers. Furthermore, it has been recently demonstrated that HARDI tractography can be used to investigate the connectivity of the brainstem reticular activating system, ${ }^{152}$ which is implicated in the pathogenesis of traumatic coma and other disorders of consciousness (see Traumatic Disorders of Consciousness section below). ${ }^{3,130}$ 
Many of the methodological limitations, confounders, and pitfalls pertaining to HARDI tractography analyses have been discussed above in the sections on DTI and diffusion tensor tractography. However, it is also important to emphasize that even with its improved resolution for detecting crossing fibers, HARDI tractography methods are still susceptible to errors introduced by uncertainty at sites of fiber crossing. Just because HARDI incorporates multidirectional diffusion data into its tractography algorithm does not mean that the tracts passing through each voxel are necessarily traveling in the anatomically correct direction. In deterministic, streamline tractography techniques, a fiber tract may take a "wrong turn" at any voxel that contains a multidirectional ODF. There is thus inherent uncertainty in any deterministic tractography analysis that may confound the white matter connectivity findings. A major advance in tractography analysis that attempts to minimize this confounder was made by Behrens and colleagues in 2003 with the development of probabilistic tractography. ${ }^{153,154}$ The probabilistic model, which may be applied to both diffusion tensor and HARDI data, aims to account for the uncertainty in fiber tract direction at each voxel. As opposed to the deterministic tractography model, which creates a single "streamline" passing through adjacent voxels, the probabilistic tractography model generates a distribution of all possible streamlines that may pass through these voxels. A Monte Carlo simulation is performed in which thousands of potential fiber tracts are generated from a single seed ROI, and the neuroanatomic distribution of all possible fiber tract paths is then displayed on a structural image. If one of the fiber tracts takes a "wrong turn" at a single voxel, this aberrant fiber tract usually terminates quickly due to a lack of coherent directional diffusion in neighboring voxels. By contrast, fiber tracts that travel along pathways with coherent directional diffusion will continue to extend to their grey matter targets.

Not surprisingly, when using manually drawn ROIs, the probabilistic tractography approach generates tracts with greater intra-subject and inter-exam reproducibility than do deterministic tractography models. ${ }^{92}$ The application of probabilistic tractography to HARDI data thus provides perhaps the greatest potential for clinicians to obtain biologically valid, quantitative metrics of white matter connectivity in patients with TBI. Still, substantial work remains before these approaches are validated for clinical use. At the present time, no form of tractography analysis is included in the imaging guidelines recommended by the Federal Interagency Initiative on Common Data Elements for TBI. ${ }^{16,17}$

\section{Advanced Functional Imaging Techniques}

\section{Resting State Functional MRI (rs-fMRI)}

The observation that spontaneous, temporally correlated fluctuations in brain activity occur in functionally related brain regions at rest, and that these regions are deactivated during goal-directed cognitive tasks, has led to the concept of "resting state networks" in the human brain. These resting state networks include the "default mode network" (DMN), ${ }^{155-158}$ "salience network," 159 thalamocortical networks, ${ }^{160}$ and the "executive control network,"159 with the DMN receiving the most attention in human studies to date. While the initial description of the DMN by Shulman, Raischle and colleagues utilized PET measurements of resting cerebral blood flow and oxygen extraction, ${ }^{155,157}$ most subsequent studies utilized blood oxygen level-dependent (BOLD) resting state fMRI (rs-fMRI) to assess the functional connectivity of the DMN. ${ }^{161,162}$ In rs-fMRI studies, functional connectivity is defined by temporal correlations in the low frequency $(<0.1 \mathrm{~Hz})$, spontaneous fluctuations of the BOLD signal. ${ }^{163}$ Hence, the functional connectivity of the DMN can be investigated by analyzing BOLD fluctuations within the grey matter nodes of the DMN, which include the posterior cingulate/retrosplenial cortex, precuneus, medial prefrontal cortex, inferior parietal lobule, and hippocampal formation. ${ }^{164,165}$ From a methodological standpoint, rs-fMRI network 
connectivity can be analyzed using a variety of approaches, such as independent component analysis or seed-based (i.e. ROI) techniques. ${ }^{166-168}$

The relevance of rs-fMRI to patients with TBI is highlighted by recent studies demonstrating that rs-fMRI measurements of DMN connectivity are altered across the spectrum of mild, moderate, and severe injury (Fig. 8). ${ }^{104,169-176}$ In addition, longitudinal increases in DMN connectivity have been correlated with functional recovery, ${ }^{172}$ and the severity of DMN dysfunction has been shown to predict neurocognitive task performance. ${ }^{173}$ Moreover, functional connectivity of cortical nodes within the DMN, as measured by rs-fMRI, correlates with structural injury in the white matter pathways connecting these nodes, as measured by DTI. ${ }^{104,171,173,175}$ These correlative rs-fMRI/DTI findings suggest that the integration of advanced imaging techniques may enable individualized assessments of structural white matter disconnections and their corresponding functional alterations in grey matter.

Yet, despite growing evidence that alterations in DMN connectivity occur across the pathophysiologic spectrum of TBI, the specific nature of these DMN alterations has yet to be fully elucidated. In an rs-fMRI study of DMN connectivity in patients in the chronic stage of severe TBI, DMN connectivity was higher at rest and showed greater deactivation during a choice-reaction task (pressing a button corresponding to a visual stimulus) in TBI patients as compared to healthy controls. The latter finding was interpreted as reflecting an increase in cognitive effort required to perform the task, since larger DMN deactivation in healthy control subjects is associated with more cognitively demanding tasks. ${ }^{177}$ In contrast, rs-fMRI studies of DMN connectivity in patients in the subacute stage (1 to 3 weeks) of mild TBI have demonstrated a reduced number and strength of connections in multiple components of the DMN network, particularly those connecting with the posterior cingulate region. ${ }^{169,174}$ The pathophysiologic mechanisms underlying these variable changes in DMN connectivity have yet to be fully elucidated. The heterogeneous effects of TBI on DMN connectivity may be explained by the nonuniform neuroanatomic distribution of TAI (i.e. focal, multi-focal, or diffuse) in patients with varying injury severities, or by the dynamic interactions between axonal degeneration and neuronal plasticity at each stage of recovery. In addition, the conflicting DMN connectivity results may be attributable to the inherent complexity of the widely distributed DMN and its apparent role in not only resting brain activity, but also a broad range of internally directed or self-referential cognitive processes, such as autobiographical memory, theory of mind, and moral decision-making. ${ }^{164}$ Additional research is therefore needed to determine whether specific types of DMN alterations are associated with specific neurocognitive deficits in patients with TBI.

Importantly, studies by Fox, Laureys, Boly, Vanhaudenhuyse and others have begun to identify unique functional domains in the human brain that mediate different aspects of awareness, providing an important foundation for future inquiries. Evidence from FDG-PET and BOLD rs-fMRI studies suggests that the introspective, self-directed component of awareness is mediated by an "intrinsic network" of midline structures (i.e. precuneus, cingulate, and medial prefrontal cortices), whereas the environmental, externally directed component of awareness is mediated by an "extrinsic network" of lateral structures (i.e. lateral fronto-parietal cortices). ${ }^{161,168,178-180}$ This distinction between intrinsic (DMN) and extrinsic awareness networks provides a neuroanatomic framework for studying the effects of TBI on different aspects of cognition.

There are several methodologic advantages of rs-fMRI over stimulus-related and taskrelated (i.e. "active") fMRI paradigms. First, rs-fMRI has a favorable signal-to-noise profile. As Fox and Greicius explain in their review on this subject, ${ }^{166}$ the spontaneous BOLD fluctuations that are analyzed in an rs-fMRI study account for approximately $60 \%$ of the 
variance in the BOLD signal. By contrast, task-induced changes in the BOLD signal typically account for less than $20 \%$ of the variance in spontaneous BOLD fluctuations. As a result of this three-fold decline in the signal-to-noise profile, task-related fMRI studies involve repetition of tasks and averaging of data acquired over multiple epochs of data acquisition. A second advantage of the rs-fMRI technique is the ability to analyze multiple resting state networks within a single dataset, thereby reducing data acquisition time. Third, Biswal and colleagues recently demonstrated in an international, multicenter study of more than 1,000 subjects that rs-fMRI analyses are readily reproducible with high test-retest reliability. ${ }^{181}$ Finally, the rs-fMRI technique may be used to investigate resting state networks across the spectrum of states of consciousness, throughout each stage of the sleepwake cycle, and even during anesthesia. ${ }^{168,182,183}$ In contrast, task-related fMRI paradigms are inherently limited in the setting of altered consciousness, sleep, and anesthesia.

Nevertheless, there are important limitations to the rs-fMRI technique that should be considered. First, administration of sedatives may confound rs-fMRI connectivity analyses, as demonstrated by Boveroux and colleagues in their analysis of resting state networks in subjects receiving propofol. ${ }^{184}$ Second, the BOLD signal that is used as a surrogate for neural activity in rs-fMRI studies is affected by a variety of physiological parameters, including cerebral blood flow (CBF), blood volume and oxygen consumption, and therefore the BOLD signal is not a direct measure of neuronal activity. Third, the BOLD signal reflects changes in venous blood oxygenation that cannot be quantified in an absolute manner or with physiologic units; rather, changes in the BOLD signal are defined in relative terms or using statistical models. Fourth, there are potential sources of artifact in the rsfMRI signal that are not related to neuronal activity or cerebrovacular hemodynamics. These include the behavioral state of the patient (i.e. eyes closed, eyes open and inattentive, or eyes open and fixated on a visual target), ${ }^{185}$ as well as hardware noise. ${ }^{186}$ Care must therefore be taken when comparing results across cohorts that the data were acquired under similar conditions. The Common Data Elements guidelines include a discussion of rs-fMRI techniques and their application to TBI, but rs-fMRI is not included in its current protocols. ${ }^{16}$

\section{Stimulus- and Task-Related fMRI}

The discovery that BOLD signal changes can be detected in the brain during stimulus-and task-related fMRI experiments has revolutionized the study of human brain function. Since the discovery of BOLD fMRI by Kwong, Ogawa, and others in 1992, ${ }^{187,188}$ fMRI has rapidly overtaken PET, single photon emission computed tomography (SPECT), electroencephalography (EEG), and magnetoencephalography (MEG) as the most commonly used technique for evaluating brain function (Fig. 7). ${ }^{189}$ The methodological features and technical limitations of fMRI are described in Orringer, Vago, and Golby's paper on "Clinical Applications and Future Directions of Functional MRI" in this issue of Seminars in Neurology and therefore will not be reviewed here. Our focus in this section will be on the studies that have applied the BOLD fMRI technique to patients with TBI. It should also be noted that the application of the ASL technique to fMRI studies is growing rapidly and ASL fMRI may have several methodological advantages over BOLD fMRI, as discussed in the Arterial-spin Labeled Perfusion MRI section below.

Functional MRI has recently been used to identify abnormal brain activation patterns in TBI patients with a variety of neurocognitive and behavioral deficits, including memory impairment ${ }^{104,190}$ and motor dysfunction. ${ }^{191}$ Palacios and colleagues demonstrated that BOLD signal changes during an "n-back" memory paradigm correlated with working memory performance in patients in the chronic stage of severe TBI. ${ }^{104}$ In this study, working memory performance and functional activation patterns also correlated with DTI measurements of FA in associative fasciculi of the cerebral hemispheres, a finding that 
underscores the utility of multimodal integration of advanced imaging methods to elucidate the pathophysiologic basis of neurocognitive deficits after TBI. In addition to investigating the pathophysiological basis for cognitive dysfunction after TBI, fMRI has also been used to detect abnormal brain activation patterns in TBI patients without neurological impairment. For example, Mayer and colleagues found that despite near-normal performance on neurocognitive testing of attention, patients with recent mild TBI ( $<3$ weeks post-injury) experienced different patterns of activation in the dorsolateral prefrontal cortex and deactivation in the DMN during attention-demanding tasks, as compared to controls. ${ }^{192}$ Similarly, Newsome and colleagues observed in a cohort of adolescents (age 12 to 19 years) with chronic moderate-to-severe TBI that during a perspective-taking task (i.e. thinking of the self from a third-person perspective), the TBI patients experienced BOLD signal changes in regions such as the cuneus and parahippocampal gyrus that are not activated in controls. ${ }^{193}$ These differential brain activation patterns on fMRI were present despite the fact that patients exhibited normal performance scores on neurocognitive tests conducted outside the MRI scanner. Functional MRI may therefore be used to elucidate the biological mechanisms involved in neuroplasticity, as BOLD activation patterns reveal the neural pathways responsible for recovery of function after TBI.

\section{Arterial-spin Labeled (ASL) Perfusion MRI}

The development of ASL perfusion imaging by Detre and colleagues in 1992 ushered in a new era of functional neuroimaging by providing quantitative CBF measurements. ${ }^{194}$ ASL utilizes a radiofrequency pulse to "label" water protons in blood flowing through the carotid arteries by inverting their spins, thereby generating an endogenous contrast. The dissipation of the labeled spins in the distal cerebrovasculature is used as an indirect measure of CBF by subtracting a labeled tagged image from an unlabeled control image. In addition to being non-invasive, ASL perfusion imaging can be performed rapidly, ${ }^{195}$ it has high test-retest reliability, ${ }^{196}$ and it can be repeated within a single scanning session to assess changes in CBF, such as those related to the administration of stimulant medications. ${ }^{197}$ Furthermore, ASL can be used to selectively label water protons within specific intracranial and extracranial vessels, enabling focused CBF measurements within individual vascular territories. ${ }^{198}$ A major advantage of ASL is that unlike BOLD, ASL provides a direct measurement of arterial perfusion that can theoretically be quantified in absolute units of $\mathrm{CBF}(\mathrm{cc} / 100 \mathrm{gm} / \mathrm{min}$ ) if one has knowledge of the longitudinal relaxation rate of blood and tissue, as well as the labeling efficiency and arterial transit delays. The ASL signal should therefore correlate directly with neuronal activity as long as cerebrovascular autoregulation and activation-flow-coupling mechanisms are intact. ${ }^{199}$

ASL perfusion imaging studies have revealed alterations in global and regional resting CBF in patients across the spectrum of TBI severity. Kim and colleagues demonstrated that patients in the chronic stage of moderate-to-severe TBI have reduced global CBF in the resting state, as well as decreased regional perfusion in the thalamus, posterior cingulate cortex, and frontal cortex. ${ }^{200}$ Notably, reductions in thalamic perfusion correlated with the degree of thalamic atrophy, suggesting that ASL perfusion measurements may reflect the functional capacity of injured neurons within a neuronatomic region. Furthermore, in a subsequent study by the same laboratory, neuroanatomic regions with decreased resting $\mathrm{CBF}$ also had altered task-related activation during an ASL fMRI working memory paradigm. ${ }^{201}$ These findings further support the possibility that resting CBF measurements in specific cortical regions may predict the functional potential of those regions in patients with TBI. Moreover, decreased resting thalamic perfusion in patients with chronic mild TBI has been correlated with functional deficits in a broad range of neurocognitive domains, including memory, attention, and processing speed. ${ }^{202}$ Taken together, these ASL perfusion analyses in the mild, moderate, and severe TBI populations suggest that CBF measurements 
have great potential to predict functional outcomes and elucidate the pathophysiologic basis of post-traumatic neurocognitive deficits.

An important consideration when interpreting the results of an ASL study is the manner in which the radiofrequency pulse is applied to label water protons in carotid blood. Currently available labeling schemes include pulsed ASL (pASL), continuous ASL (cASL), pseudocontinuous ASL (pcASL) 203,204 and velocity-selective ASL (vSASL). ${ }^{205}$ In the pASL labeling scheme, the perfusion measurements may susceptible to physiological noise due to variable labeling of the carotid blood during the systolic or diastolic phases of the cardiac cycle. By contrast, the cASL labeling scheme reduces cardiac modulation of the ASL signal because the radiofrequency pulse may be administered over the entire cardiac cycle (i.e. during both systole and diastole). ${ }^{206}$ However, special hardware and higher specific absorption rates (SAR) are associated with cASL, ${ }^{203}$ which limits its widespread implementation. PcASL is a combined approach that uses multiple radiofrequency pulses to obtain the high labeling efficiency of pASL and a signal-to-noise ration that is comparable to that of cASL. ${ }^{203,204}$ Versions of pcASL are available from three major MR scanner manufacturers.

Another potential source of artifact affecting the ASL signal is variability in the transit time for labeled blood to flow from the tagging region in the carotid arteries to the downstream sites of image acquisition, such as in the setting of extracranial or intracranial cerebrovacular stenosis. VsASL approaches attempt to compensate for arterial transit delays by labeling on the basis of velocity rather than spatial location. ${ }^{205}$ Additional considerations are the lesser degree of ASL signal change during functional activation as compared to the corresponding BOLD signal change, as well as the lesser amount of brain coverage (i.e. lower number of slices that are imaged) during an ASL study as compared to a BOLD study. ${ }^{199}$ Because of the technique's reliance on relatively small signal differences, it is important to review the raw control and tag images to assess for false positive findings that may be caused by artifacts (e.g. EEG leads, patient motion during the scan). ASL perfusion imaging is currently listed in Tier 2 of the imaging protocols recommended in the Common Data Elements guidelines for 3T Siemens scanners using pASL. ${ }^{16}$ However, there is currently a lack of consensus regarding the best labeling scheme, though many researchers prefer pcASL for reasons stated above. ${ }^{207}$ For commercially available ASL sequences, protocols need to be optimized (e.g. post-labeling delay) according to age and disease. Reproducibility of results across scanner manufacturers using a standardized labeling scheme has yet to be studied rigorously.

\section{Positron Emission Tomography (PET)}

The development of ${ }^{18}$ F-fluorodeoxy-glucose (FDG)-PET by Reivich, Greenberg and colleagues led to major new insights in human brain function over the past three decades. ${ }^{208,209}$ FDG-PET provides a measurement of the glucose metabolism within neuronal mitochondria and thus may be used to measure both resting and task-related changes in brain metabolism. Many of the insights into TBI pathophysiology that have been made by FDG-PET imaging studies have been reviewed by Dubroff and Newberg in a previous issue of Seminars in Neurology. ${ }^{18}$ Yet, despite the important discoveries generated by FDG-PET, its cost, limited availability, and radiation exposure have diminished its use in recent years, especially with the growing availability of BOLD and ASL fMRI.

Nevertheless, as discussed above, Shulman, Raichle and colleagues used oxygen-based PET techniques to make several of the initial discoveries about the DMN. Furthermore, Schiff and Laureys' laboratories have used FDG-PET and ${ }^{15} \mathrm{O}$-radiolabeled water PET over the past decade to generate novel insights into the pathophysiological mechanisms underlying traumatic disorders of consciousness. ${ }^{180,210-213}$ These PET studies are discussed below in the section on Traumatic Disorders of Consciousness. Of note, PET techniques are discussed 
in the Common Data Element guidelines but are not included in the formally recommended protocols. ${ }^{16}$ For further details regarding the methodologic basis of PET imaging, please see Tarun Singhal's article on "Positron Emission Tomography Applications in Clinical Neurology" in the current issue of Seminars in Neurology.

\section{Magnetic Resonance Spectroscopy (MRS)}

MRS assesses the biochemical profile of a neuroanatomic region within the brain. The relative concentrations of metabolites are represented as a series of peaks along a spectrum, and abnormal ratios between the peaks may be diagnostic of metabolic dysfunction. The development of multivoxel spectroscopy (chemical shift imaging) also allows the clinician to investigate the distribution of metabolite concentrations over a larger expanse of neuroanatomic territory. As with PET, the methodological basis of MRS and its application to the care of patients with TBI have been reviewed in a prior issue of Seminars in Neurology.${ }^{18,214}$ For the purpose of this review, we wish to highlight one recent study that demonstrated the potential utility of MRS as a prognostic tool in TBI. In an analysis of 43 patients with TBI who were unable to follow simple commands, MRS performed at a mean of 24 days post-injury predicted functional outcomes at 1 year, as determined by a dichotomized Glasgow Outcome Scale score. ${ }^{78}$ Specifically, NAA/Cr ratios in the pons, thalamus, and insula predicted an unfavorable 1-year outcome with $75 \%$ sensitivity and $75 \%$ specificity. Furthermore, when these MRS measurements were combined with DTI measurements of supratentorial FA, the combined predictive value of MRS and DTI for unfavorable outcome increased to $86 \%$ sensitivity and $97 \%$ specificity. These findings suggest that not only does MRS provide prognostically useful data in the early subacute stage of TBI, but that the integration of data from multiple advanced imaging techniques has great potential to improve prognostication. MRS is listed in tier 2 of the Common Data Element guidelines. ${ }^{16}$

\section{Special Considerations}

\section{TBI in Military Personnel}

TBI is considered the "signature injury" of the Afghanistan and Iraq wars, accounting for the majority of all combat casualties. ${ }^{215}$ More than 300,000 military personnel are estimated to have experienced a TBI since the initiation of Operation Enduring Freedom and Operation Iraqi Freedom. ${ }^{2}$ Furthermore, soldiers are surviving TBI at a higher rate than in past wars because of improvements in body armor and improved access to acute life-saving therapies. ${ }^{215,216}$ Thus, more soldiers are returning home with the neurological sequellae of severe TBI, which often involves impaired levels of consciousness. ${ }^{216-218}$ As a result, the United States Department of Veterans Affairs has instituted "Emerging Consciousness" programs throughout the United States to facilitate the rehabilitation of military personnel who have survived a traumatic coma. Between 2007 and 2009, 67 of 97 military personnel enrolled in these programs ultimately regained consciousness. ${ }^{219}$

As in past wars, blunt and missile head trauma account for a significant percentage of TBIrelated neurological injury, but the exposure of military personnel to blast-related injuries from improvised explosive devices has created new challenges in the care of military personnel. ${ }^{217}$ During a blast explosion, a series of mechanical forces are exerted upon the skull in rapid succession, beginning with an initial shock wave that gives rise to a supersonic blast wind, followed by a more prolonged phase of negative pressure. ${ }^{220}$ Although the mechanism(s) of brain injury during a blast are incompletely understood, it is believed that the initial shock wave directly impacts the brain by penetrating the intracranial vault via the acoustic canals, optic canals, and/or foramen magnum. ${ }^{221}$ Upon reaching the intracranial vault, the energy of the shock wave is transmitted to the brain parenchyma and 
cerebrovasculature, potentially resulting in TAI, cerebral edema, intracerebral hemorrhage, subarachnoid hemorrhage, arterial vasospasm, and/or pseudoaneurysm formation. ${ }^{215,222}$ Even when conventional neuroimaging methods fail to detect focal brain injury, soldiers exposed to blast waves often report symptoms suggestive of post-concussive syndrome, including memory impairment, inattention, dizziness, and headaches. ${ }^{215}$

Yet, significant controversy remains regarding the precise pathophysiologic effects of blast waves on the human brain, especially since conventional neuroimaging techniques are often unable to detect the effects of blast injury. ${ }^{76,223}$ Even advanced imaging studies utilizing DTI initially failed to identify alterations in white matter FA in military veterans with a history of mild-to-moderate blast-related TBI. ${ }^{224}$ This difficulty in detecting structural brain injury in patients exposed to blasts is especially problematic for military personnel, since the high rate of neurocogntive sequelae from concurrent post-traumatic stress disorder may confound the assessment of blast-related deficits. ${ }^{225}$ Yet, in the largest study performed to date in soldiers exposed to blast injuries, Mac Donald and colleagues demonstrated that DTI measurements of white matter anisotropy (relative anisotropy was the quantitative metric used in this study) are indeed able to detect structural abnormalities in the brains of military personnel with TBI due to blast injury. ${ }^{76}$ Although none of the soldiers in this study had isolated blast injury (all experienced concurrent blunt trauma, motor vehicle accident, or fall), the cohort with blast injury and a clinical diagnosis of mild TBI had more areas of abnormal anisotropy than a control cohort of soldiers who had been exposed to blast but who did not have a diagnosis of TBI. Abnormal anisotropy measurements were detected in the middle cerebellar peduncle and orbitofrontal white matter in the blast TBI cohort as compared to the blast without TBI cohort. In addition, follow-up DTI scans demonstrated persistent, evolving changes in these white matter regions in the blast TBI cohort. A recent study by Bazarian and colleagues involving 52 veterans of the Afghanistan and Iraq wars demonstrated that blast exposure was also associated with abnormalities in white matter anisotropy. ${ }^{223}$ These studies in military personnel provide proof of principle that advanced imaging techniques, particularly DTI, may enhance the detection of blast-related brain injury and may ultimately enable clinicians to distinguish the effects of blast TBI from posttraumatic stress disorder. Of note, MRI scanners were recently deployed to Kandahar Airfield Base, Bagram Air Base, and Camp Bastion in Afghanistan, marking the first time in history that MRI scanners have been deployed in a theater of war. ${ }^{87}$

\section{Sports Concussion}

The neurological sequellae associated with sports concussion have been recognized for decades, but concussion-related neurological injury has received increasing attention in recent years for two main reasons. First, histopathological evidence of chronic traumatic encephalopathy (CTE), a progressive tauopathy with neuropathologic features that are distinct from other degenerative diseases such as Alzheimer's disease, has been reported in the brains of amateur and professional athletes who sustained concussions while competing in football, boxing, soccer, or professional wrestling. ${ }^{226}$ Second, advanced neuroimaging techniques are for the first time providing clinicians with a window into the structural and functional basis for post-concussive neurocognitive deficits. A multitude of advanced imaging studies using each of the techniques described above have been used to identify brain injury in patients with neurocognitive deficits caused by sports concussion. ${ }^{227}$ In a particularly noteworthy study, Zhang and colleagues performed rs-fMRI analyses of the DMN immediately before and after physical exertion in patients with mild TBI (mean 10 days post-injury). ${ }^{170}$ In these college student-athletes, there was no difference in DMN connectivity between concussed athletes and controls in the pre-exertion DMN analysis, but there was a significant decline in DMN connectivity in the concussed athletes after exertion. These findings may help to explain the commonly observed phenomena that post-concussive 
symptoms are exacerbated by exertion, while also providing support for current recommendations in the American Academy of Neurology Practice Parameter on Concussion that athletes should be asymptomatic both at rest and after exertion before returning to play. ${ }^{228}$ Another rs-fMRI study identified persistent alterations in DMN connectivity in concussed college athletes who had been asymptomatic for at least 24 hours and medically cleared to return to aerobic activity by a physician. ${ }^{169}$ The possibility that advanced imaging techniques may detect alterations in brain function beyond the time of symptom resolution suggests that advanced imaging may ultimately be incorporated into future guidelines regarding safe return to play.

Nevertheless, there is still no clearly defined set of acute imaging findings in a concussed athlete that predict a rapid recovery versus the development of persistent post-concussive symptoms ( $>3$ months), which occur in up to $30 \%$ of concussed individuals. ${ }^{229}$ Further complicating the effort to use advanced imaging techniques to detect concussion-related brain injuries is the extensive heterogeneity in the neuroantaomic distribution of injury, an observation that likely reflects the variability in injury mechanisms. While many DTI studies have identified altered white matter FA in the brains of concussed athletes, a DTI study by Cubon and colleagues using the TBSS analytic technique found abnormalities in white matter mean diffusivity but not FA. ${ }^{101}$ Furthermore, Zhang and colleagues did not identify a consistent pattern of structural or functional brain abnormalities in concussed athletes using either DTI or fMRI. ${ }^{230}$ It therefore remains to be determined which advanced imaging technique and which quantitative metric of structural and/or functional brain injury will be most sensitive and specific for detecting concussion-related brain injury and predicting recovery.

Just as additional research is needed to clarify the mechanisms and patterns of injury that occur in the early stages of sports concussion, the pathophysiological mechanisms that lead from concussion to CTE have yet to be determined. Recent evidence suggests that genetic factors, such as the apolipoprotein E epsilon 4 allele, may play a contributory role in determining whether a concussed athlete will develop chronic symptoms and/or CTE. ${ }^{231}$ Thus, advanced imaging data may need to be integrated with genomic data to optimize the accuracy of prognostication and the development of new therapies.

\section{Traumatic Disorders of Consciousness}

The use of advanced neuroimaging techniques in patients with traumatic disorders of consciousness (DOC) has been motivated by several factors. First, bedside examination of patients believed to be in VS is associated with a misdiagnosis rate of up to 43\% $232-234$ when compared to standardized neurocognitive evaluation with the Coma Recovery ScaleRevised (CRS-R) developed by Giacino and colleagues. ${ }^{235}$ This alarming statistic may be attributed to impairments in visual, auditory, motor, or language function that limit the patient's ability to interact with the environment despite intact cognition. Additionally, Schnakers and colleagues demonstrated that certain elements of the CRS-R have increased sensitivity for detecting purposeful behavior as compared to a typical bedside exam (i.e. testing the patient's ability to perform gaze tracking with a mirror as opposed to the examiner's finger). ${ }^{234}$ Second, advanced imaging techniques have begun to provide extraordinary insights into residual cognitive function in patients with DOC that could not be otherwise be revealed by bedside examination or conventional neuroimaging. Third, there is an urgent need for diagnostic techniques to identify patients who may benefit from pharmacologic and electrophysiologic therapies aimed at restoring consciousness. Indeed, Giacino, Whyte and colleagues recently demonstrated in a placebo-controlled, multiinstitutional study that amantadine accelerates the pace of recovery of consciousness in patients with traumatic DOC. ${ }^{236}$ There are also a growing number of reports of paradoxical "awakenings" produced by the GABA-agonist zolpidem. ${ }^{237}$ Moreover, Schiff, Giacino, and 
colleagues demonstrated that thalamic deep brain stimulation improved behavioral responsiveness in a patient with chronic minimally conscious state (MCS). ${ }^{238}$ The efficacy of these therapies in some patients with DOC provides an ethical basis to use advanced imaging techniques to search for residual neural network activity that may have the potential to support meaningful neurological recovery. ${ }^{239}$

One of several groundbreaking studies to demonstrate that advanced imaging techniques may identify brain activity that evades detection by bedside examination was published by Owen and colleagues in $2006 .{ }^{240}$ In this study, a 23-year-old woman in a traumatic VS caused by a motor vehicle accident was asked to perform a motor imagery task (imagining playing tennis) and a spatial imagery task (imagining walking through her home) while in the MRI scanner. BOLD fMRI activation of the supplementary motor area was observed during the motor imagery task, and activation of the parahippocampal gyrus, posterior parietal cortex, and lateral premotor cortex was observed during the spatial imagery task, which were the same fMRI activation patterns observed in healthy control subjects. For the patient, these fMRI responses were present despite the absence of any evidence of awareness of self or environment on detailed neurological examination by a multidisciplinary team. Subsequent fMRI studies using various motor imagery and spatial imagery paradigms have reproduced this finding, further demonstrating that some patients diagnosed with VS or MCS have fMRI activation patterns that are similar to those of healthy control subjects. In the largest such study to date, Monti and colleagues found that 5 of 54 patients with DOC (23 in VS and 31 in MCS; 33 with TBI) could willfully modulate their brain responses to motor and spatial imagery paradigms (all five patients had TBI as the etiology of the DOC). Furthermore, a single subject in traumatic VS was able to consistently communicate "yes" and "no" responses by performing specific imagery tasks while in the MRI scanner. ${ }^{241}$ These findings build upon prior observations by Schiff and colleagues that patients with traumatic disorders of consciousness may possess regions of preserved cerebral metabolism, or "islands" of neural network activity, which persist despite catastrophic brain injury. ${ }^{212}$ In total, these studies suggest that advanced neuroimaging techniques may provide evidence of conscious awareness and behavioral responsiveness that evades detection on bedside examination, thus challenging classical concepts about consciousness and raising questions as to whether advanced imaging data should be incorporated into standard clinical definitions of DOC. ${ }^{242}$

A series of fMRI and PET studies by Boly, Schiff, Coleman, and others have also demonstrated that auditory stimuli and language paradigms may identify activation of periSylvian language networks in patients with DOC who do not appear to express or understand language at the bedside. ${ }^{213,243-246}$ One such study by Coleman and colleagues found that language-related fMRI activation patterns correlated with the degree of functional recovery 6 months after the fMRI scan. ${ }^{244}$ The application of advanced imaging analysis to the study of patients with traumatic DOC has also begun to shed light on the pathophysiological profiles that are associated with each diagnosis along the clinical spectrum of DOC. Several fMRI and PET studies have shown that VS is associated with decreased function within the posterior nodes of the DMN, such as the precuneus and posterior cingulate cortex. ${ }^{247-249}$ Accordingly, recovery of metabolic activity and/or perfusion within the precuneus and posterior cingulate appears to correlate with recovery of conscious awareness in patients with VS. ${ }^{247,248}$ In MCS patients, Liu and colleagues used ASL to demonstrate relative preservation of CBF in precuneus/posterior cingulate but relatively decreased blood flow in the anterior nodes of the DMN (i.e. medial prefrontal cortex). ${ }^{250}$ Taken together, these ASL, fMRI, and PET studies suggest that the transition from VS to MCS appears to be associated with reintegration of the posterior cingulate/ precuneus into the DMN, whereas emergence from MCS may be associated with reintegration of the medial prefrontal cortex into the DMN. Indeed, Vanhaudenhuyse and 
colleagues recently demonstrated that the degree of DMN connectivity determined by rsfMRI correlated with a patient's level of consciousness, as determined by the CRS-R. ${ }^{248}$ For further discussion of the advanced neuroimaging studies that have shed new light on the structural and functional basis of traumatic DOC, we refer the reader to a recent review on this subject by Laureys and Schiff. ${ }^{251}$

As clinicians consider incorporating advanced imaging techniques into the diagnostic and prognostic evaluation of patients with traumatic DOC, it is important to note that in addition to all of the modality-specific limitations discussed above, there is also a more general consideration in the DOC population that the sensitivity and specificity of these techniques for detecting evidence of conscious awareness remains unknown pending large, multi-center studies. For example, a recent study by Bardin and colleagues found that the commonly used general linear model-based univariate approach to fMRI analysis may not be as sensitive at detecting BOLD fMRI activation in patients with DOC as a newly developed multivariate pattern analysis classification approach. ${ }^{252}$ Furthermore, there are a myriad of potential confounders that are particularly relevant in this population, including delayed responses that evade detection by brief fMRI paradigms ${ }^{253}$ and state changes (i.e. transitions from wakefulness to drowsiness to sleep) that may occur while the patients is in the scanner. These methodological issues and potential confounders suggest that further research is needed before fMRI can become part of the routine assessment of patients with traumatic DOC, especially in light of the profound implications of these fMRI results for the patient and the family.

Finally, although major advances have been made in recent years in understanding the structural and functional basis of awareness in patients with traumatic DOC, current understanding about arousal (wakefulness) lags far behind. Arousal is a critical component of consciousness, without which awareness is not possible. The current lack of understanding about the pathophysiological mechanisms that cause altered arousal in traumatic DOC can be explained by the inability of conventional imaging tools to identify the complex neuroanatomic connectivity of the ascending reticular activating system (ARAS). Since the discovery of the ARAS by Moruzzi and Magoun in $1949,{ }^{254}$ the vast majority of structural and functional analyses of this arousal network has been performed in animal models, ${ }^{255}$ which may not be directly relevant to patients with traumatic DOC because of the unique features of human consciousness. Kinomura and colleagues confirmed in humans the presence of functional connectivity between the dorsal midbrain and thalamus in a PET study of attention-demanding tasks in healthy control subjects. ${ }^{256}$ Parvizi and Damasio also generated key insights into the specific brainstem nuclei that are essential to arousal, and hence consciousness. ${ }^{115}$ Yet, the 3-dimensional connectivity of the ARAS and its role in traumatic DOC have yet to be fully elucidated in human patients.

For clinicians to accurately determine a patient's chances of recovery, advanced imaging techniques must be developed to delineate the structural and functional integrity of not only the hemispheric pathways that mediate awareness, but also the ARAS pathways mediating arousal. This goal is fundamentally important to predicting recovery after traumatic coma, because the human ARAS appears to contain redundant connectivity between the brainstem and diencephalon, which may enable recovery of arousal when some, but not all components of the ARAS network are disrupted. ${ }^{115}$ Recently, HARDI tractography was used to generate preliminary insights into the neuroanatomic pathways linking the brainstem arousal nuclei to their thalamic, hypothalamic, and basal forebrain targets in the ex vivo and in vivo human brain. ${ }^{152}$ Yet, the HARDI sequence utilized in the in vivo analysis in this study required a long duration of acquisition (30 minutes) to acquire data with high angular and spatial resolution (120 diffusion directions, $b$ value $=4000 \mathrm{sec} / \mathrm{mm}^{2}$, and voxel size $2 \times 2 \times 2 \mathrm{~mm}$ ), which may not be feasible in subjects with DOC who are unable to follow commands or 
remain still for long periods of time. Thus, increasing the speed of HARDI data acquisition will be necessary before these advanced techniques are brought to the bedside.

\section{Future Directions - Multimodal Data Integration and Visualization}

Major methodological hurdles remain and significant additional research is needed before many of the advanced imaging techniques discussed in this review will be incorporated into clinical care. Furthermore, automated analysis tools will need to be developed and validated to enable rapid, robust and reproducible interpretation of advanced imaging data at the point of care, so that clinicians are able to use these data to inform clinical decisions.

Nevertheless, just as conventional MRI has been shown to be superior to CT in providing clinically useful diagnostic and prognostic data for patients with TBI, there is a rapidly growing body of evidence that advanced imaging techniques will surpass conventional MRI in their clinical utility. The challenge that will therefore confront clinicians in coming years is how to best incorporate data from these novel techniques into clinical practice. The results of advanced imaging techniques will only be useful if they are organized into readily interpretable, easily visualizable data elements. Just as the genomic revolution spawned the development of new data analysis and visualization tools, ${ }^{257}$ there is a similar need for such tools with the advent of large-scale neural network modeling 258 and the recent initiation of the NIH-funded Human Connectome Project (see www.humanconnectomeproject.org and www.humanconnectome.org).

One approach to optimizing the analysis and visualization of neural network data has been developed by Sporns and Hagmann, who independently coined the term "connectomics" in $2005{ }^{259,260}$ In this approach, quantitative metrics of network connectivity, such as the clustering coefficient (density of connections between neighboring network nodes) and the path length (a measure of the distance between nodes in the network), are used to generate a variety of connectivity graphs, such as a connection matrix. ${ }^{258}$ Another approach to network modeling was recently proposed by Irimia and colleagues, who developed a "connectogram" to visually display the entirety of a patient's hemispheric connectivity data in a single image. The connectogram technique has been used to display cortical connectivity in healthy human subjects, ${ }^{261}$ as well as disruptions in cortical pathways in human subjects with TBI. ${ }^{262} \mathrm{~A}$ key advantage of the connectogram technique from the standpoint of clinical utility is not just its ability to display a large amount of connectivity data in a single image, but also its flexibility to integrate data from multiple advanced imaging modalities.

Ultimately, clinicians must play an active role in shaping the manner in which data is visually represented to them. This effort will be especially important when one considers that no single technique is likely to provide all of the diagnostic and prognostic data that are needed to make well informed clinical decisions. Rather, the studies discussed in this review demonstrate that multiple advanced imaging techniques have the potential to improve the care of patients with TBI. Accordingly, clinicians will need analysis and visualization tools that integrate multimodal advanced imaging data to optimize their clinical utility. Given the rapid pace of progress in recent years, we can expect that advanced imaging techniques will soon provide clinicians with the tools they need to improve the accuracy of diagnosis and prognosis for the many civilians and military personnel with TBI. Most importantly, advanced imaging tools are likely to play a key role in guiding the development of novel therapies aimed at improving outcomes for patients with this common and often devastating disease.

\section{Acknowledgments}

This work was supported by the National Institutes of Health (R25NS065743 and P41RR14075) and the Center for Integration of Medicine \& Innovative Technology (Boston, MA). 


\section{References}

1. Faul, M.; Xu, L.; Wald, MM.; Coronado, VG. Traumatic brain injury in the United States: emergency department visits, hospitalizations and deaths 2002-2006. Atlanta (GA): Centers for Disease Control and Prevention, National Center for Injury Prevention and Control; 2010.

2. Tanielian, TL.; Jaycox, L. Rand Corporation. Invisible wounds of war: psychological and cognitive injuries, their consequences, and services to assist recovery. Santa Monica, CA: RAND; 2008. p. xliiip. 453

3. Ommaya AK, Gennarelli TA. Cerebral concussion and traumatic unconsciousness. Correlation of experimental and clinical observations of blunt head injuries. Brain. 1974; 97(4):633-654.

[PubMed: 4215541]

4. Shaw NA. The neurophysiology of concussion. Prog Neurobiol. 2002; 67(4):281-344. [PubMed: 12207973]

5. Ropper AH, Gorson KC. Clinical practice. Concussion N Engl J Med. 2007; 356(2):166-172.

6. Gentry LR. Imaging of closed head injury. Radiology. 1994; 191(1):1-17. [PubMed: 8134551]

7. Gentry LR, Godersky JC, Thompson B. MR imaging of head trauma: review of the distribution and radiopathologic features of traumatic lesions. AJR Am J Roentgenol. 1988; 150(3):663-672. [PubMed: 3257624]

8. Adams JH, Doyle D, Ford I, et al. Diffuse axonal injury in head injury: definition, diagnosis and grading. Histopathology. 1989; 15(1):49-59. [PubMed: 2767623]

9. Strich SJ. Diffuse degeneration of the cerebral white matter in severe dementia following head injury. J Neurol Neurosurg Psychiatry. 1956; 19(3):163-185. [PubMed: 13357957]

10. Strich SJ. Shearing of Nerve Fibers as a Cause of Brain Damage due to Head Injury: A Pathological Study of Twenty Cases. Lancet. 1961; 2:443-448.

11. Blumbergs PC, Scott G, Manavis J, et al. Staining of amyloid precursor protein to study axonal damage in mild head injury. Lancet. 1994; 344(8929):1055-1056. [PubMed: 7523810]

12. Blumbergs PC, Scott G, Manavis J, et al. Topography of axonal injury as defined by amyloid precursor protein and the sector scoring method in mild and severe closed head injury. $\mathbf{J}$ Neurotrauma. 1995; 12(4):565-572. [PubMed: 8683607]

13. Gennarelli TA, Thibault LE, Graham DI. Diffuse Axonal Injury: An Important Form of Traumatic Brain Damage. Neuroscientist. 1998; 4:202-215.

14. Maxwell WL, Povlishock JT, Graham DL. A mechanistic analysis of nondisruptive axonal injury: a review. J Neurotrauma. 1997; 14(7):419-440. [PubMed: 9257661]

15. Adams JH, Graham DI, Jennett B. The neuropathology of the vegetative state after an acute brain insult. Brain. 2000; 123(7):1327-1338. [PubMed: 10869046]

16. Haacke EM, Duhaime AC, Gean AD, et al. Common data elements in radiologic imaging of traumatic brain injury. J Magn Reson Imaging. 2010; 32(3):516-543. [PubMed: 20815050]

17. Duhaime AC, Gean AD, Haacke EM, et al. Common data elements in radiologic imaging of traumatic brain injury. Arch Phys Med Rehabil. 2010; 91(11):1661-1666. [PubMed: 21044709]

18. Dubroff JG, Newberg A. Neuroimaging of traumatic brain injury. Semin Neurol. 2008; 28(4):548557. [PubMed: 18843581]

19. Weiss N, Galanaud D, Carpentier A, Naccache L, Puybasset L. Clinical review: Prognostic value of magnetic resonance imaging in acute brain injury and coma. Crit Care. 2007; 11(5):230. [PubMed: 17980050]

20. Marshall LF, Marshall SB, Klauber MR, et al. A new classification of head injury based on computerized tomography. J Neurosurg. 1991; 75:S14-S20.

21. Maas AI, Hukkelhoven CW, Marshall LF, Steyerberg EW. Prediction of outcome in traumatic brain injury with computed tomographic characteristics: a comparison between the computed tomographic classification and combinations of computed tomographic predictors. Neurosurgery. 2005; 57(6):1173-1182. [PubMed: 16331165]

22. Murray GD, Butcher I, McHugh GS, et al. Multivariable prognostic analysis in traumatic brain injury: results from the IMPACT study. J Neurotrauma. 2007; 24(2):329-337. [PubMed: 17375997] 
23. Steyerberg EW, Mushkudiani N, Perel P, et al. Predicting outcome after traumatic brain injury: development and international validation of prognostic scores based on admission characteristics. PLoS medicine. 2008; 5(8):e165. [PubMed: 18684008]

24. Perel P, Arango M, Clayton T, et al. Predicting outcome after traumatic brain injury: practical prognostic models based on large cohort of international patients. BMJ. 2008; 336(7641):425-429. [PubMed: 18270239]

25. Nelson DW, Nystrom H, MacCallum RM, et al. Extended analysis of early computed tomography scans of traumatic brain injured patients and relations to outcome. J Neurotrauma. 2010; 27(1):5164. [PubMed: 19698072]

26. Yuh EL, Cooper SR, Ferguson AR, Manley GT. Quantitative CT improves outcome prediction in acute traumatic brain injury. J Neurotrauma. 2012; 29(5):735-746. [PubMed: 21970562]

27. Smits M, Dippel DW, de Haan GG, et al. External validation of the Canadian CT Head Rule and the New Orleans Criteria for CT scanning in patients with minor head injury. JAMA. 2005; 294(12):1519-1525. [PubMed: 16189365]

28. Firsching R, Woischneck D, Diedrich M, et al. Early magnetic resonance imaging of brainstem lesions after severe head injury. J Neurosurg. 1998; 89(5):707-712. [PubMed: 9817405]

29. Paterakis K, Karantanas AH, Komnos A, Volikas Z. Outcome of patients with diffuse axonal injury: the significance and prognostic value of MRI in the acute phase. J Trauma. 2000; 49(6): 1071-1075. [PubMed: 11130491]

30. Gentry LR, Godersky JC, Thompson B, Dunn VD. Prospective comparative study of intermediatefield MR and CT in the evaluation of closed head trauma. AJR Am J Roentgenol. 1988; 150(3): 673-682. [PubMed: 3257625]

31. Lee $\mathrm{H}$, Wintermark M, Gean AD, et al. Focal lesions in acute mild traumatic brain injury and neurocognitive outcome: CT versus 3T MRI. J Neurotrauma. 2008; 25(9):1049-1056. [PubMed: 18707244]

32. Skandsen T, Kvistad KA, Solheim O, et al. Prognostic value of magnetic resonance imaging in moderate and severe head injury: a prospective study of early MRI findings and one-year outcome. J Neurotrauma. 2011; 28(5):691-699. [PubMed: 21401308]

33. Lagares A, Ramos A, Perez-Nunez A, et al. The role of MR imaging in assessing prognosis after severe and moderate head injury. Acta neurochirurgica. 2009; 151(4):341-356. [PubMed: 19224121]

34. Betz J, Zhuo J, Roy A, Shanmuganathan K, Gullapalli RP. Prognostic value of diffusion tensor imaging parameters in severe traumatic brain injury. J Neurotrauma. 2012; 29(7):1292-1305. [PubMed: 22364596]

35. Henry LC, Tremblay J, Tremblay S, et al. Acute and chronic changes in diffusivity measures after sports concussion. J Neurotrauma. 2011; 28(10):2049-2059. [PubMed: 21864134]

36. Schlaug G, Siewert B, Benfield A, Edelman RR, Warach S. Time course of the apparent diffusion coefficient (ADC) abnormality in human stroke. Neurology. 1997; 49(1):113-119. [PubMed: 9222178]

37. Liu AY, Maldjian JA, Bagley LJ, Sinson GP, Grossman RI. Traumatic brain injury: diffusionweighted MR imaging findings. AJNR Am J Neuroradiol. 1999; 20(9):1636-1641. [PubMed: 10543633]

38. Muccio CF, De Simone M, Esposito G, et al. Reversible post-traumatic bilateral extensive restricted diffusion of the brain. A case study and review of the literature. Brain Inj. 2009; 23(5): 466-472. [PubMed: 19408169]

39. Ommaya AK. Head injury mechanisms and the concept of preventive management: a review and critical synthesis. J Neurotrauma. 1995; 12(4):527-546. [PubMed: 8683604]

40. Scheid R, Preul C, Gruber O, Wiggins C, von Cramon DY. Diffuse axonal injury associated with chronic traumatic brain injury: evidence from T2*-weighted gradient-echo imaging at $3 \mathrm{~T}$. AJNR Am J Neuroradiol. 2003; 24(6):1049-1056. [PubMed: 12812926]

41. Tong KA, Ashwal S, Holshouser BA, et al. Diffuse axonal injury in children: clinical correlation with hemorrhagic lesions. Ann Neurol. 2004; 56(1):36-50. [PubMed: 15236400]

42. Geurts BH, Andriessen TM, Goraj BM, Vos PE. The reliability of magnetic resonance imaging in traumatic brain injury lesion detection. Brain Inj. 2012 
43. Yanagawa Y, Tsushima Y, Tokumaru A, et al. A quantitative analysis of head injury using T2*weighted gradient-echo imaging. J Trauma. 2000; 49(2):272-277. [PubMed: 10963538]

44. Scheid R, Walther K, Guthke T, Preul C, von Cramon DY. Cognitive sequelae of diffuse axonal injury. Arch Neurol. 2006; 63(3):418-424. [PubMed: 16533969]

45. Niogi SN, Mukherjee P, Ghajar J, et al. Extent of microstructural white matter injury in postconcussive syndrome correlates with impaired cognitive reaction time: a 3T diffusion tensor imaging study of mild traumatic brain injury. AJNR Am J Neuroradiol. 2008; 29(5):967-973. [PubMed: 18272556]

46. Reichenbach JR, Venkatesan R, Schillinger DJ, Kido DK, Haacke EM. Small vessels in the human brain: MR venography with deoxyhemoglobin as an intrinsic contrast agent. Radiology. 1997; 204(1):272-277. [PubMed: 9205259]

47. Haacke EM, Xu Y, Cheng YC, Reichenbach JR. Susceptibility weighted imaging (SWI). Magn Reson Med. 2004; 52(3):612-618. [PubMed: 15334582]

48. Tong KA, Ashwal S, Holshouser BA, et al. Hemorrhagic shearing lesions in children and adolescents with posttraumatic diffuse axonal injury: improved detection and initial results. Radiology. 2003; 227(2):332-339. [PubMed: 12732694]

49. Greenberg SM, Vernooij MW, Cordonnier C, et al. Cerebral microbleeds: a guide to detection and interpretation. Lancet Neurol. 2009; 8(2):165-174. [PubMed: 19161908]

50. Cordonnier C, Al-Shahi Salman R, Wardlaw J. Spontaneous brain microbleeds: systematic review, subgroup analyses and standards for study design and reporting. Brain. 2007; 130(8):1988-2003. [PubMed: 17322562]

51. Scheid R, Ott DV, Roth H, Schroeter ML, von Cramon DY. Comparative magnetic resonance imaging at 1.5 and 3 Tesla for the evaluation of traumatic microbleeds. J Neurotrauma. 2007; 24(12):1811-1816. [PubMed: 18159992]

52. Kammond, KE.; Lupo, JM.; Xu, D., et al. Microbleed Detection in Traumatic Brain Injury at $3 \mathrm{~T}$ and 7T: Comparing 2D and 3D Gradient-Recalled Echo (GRE) Imaging with SusceptibilityWeighted Imaging (SWI). ISMRM 17th Scientific Meeting \& Exhibition; Honolulu, HI, USA. 2009.

53. Nandigam RN, Viswanathan A, Delgado P, et al. MR imaging detection of cerebral microbleeds: effect of susceptibility-weighted imaging, section thickness, and field strength. AJNR Am J Neuroradiol. 2009; 30(2):338-343. [PubMed: 19001544]

54. Gregoire SM, Chaudhary UJ, Brown MM, et al. The Microbleed Anatomical Rating Scale (MARS): reliability of a tool to map brain microbleeds. Neurology. 2009; 73(21):1759-1766. [PubMed: 19933977]

55. Cordonnier C, Potter GM, Jackson CA, et al. improving interrater agreement about brain microbleeds: development of the Brain Observer MicroBleed Scale (BOMBS). Stroke. 2009; 40(1):94-99. [PubMed: 19008468]

56. Stejskal EO. Use of spin echoes in a pulsed magnetic-field gradient to study anisotropic, restricted diffusion and flow. J Chem Phys. 1965; 43(10):3597-3603.

57. Pierpaoli C, Basser PJ. Toward a quantitative assessment of diffusion anisotropy. Magn Reson Med. 1996; 36(6):893-906. [PubMed: 8946355]

58. Basser PJ, Pierpaoli C. Microstructural and physiological features of tissues elucidated by quantitative-diffusion-tensor MRI. J Magn Reson B. 1996; 111(3):209-219. [PubMed: 8661285]

59. Basser PJ, Mattiello J, Le Bihan D. Estimation of the Effective Self-Diffusion Tensor from the NMR Spin Echo. J Magn Reson B. 1994; 103(3):247-254. [PubMed: 8019776]

60. Basser PJ, Jones DK. Diffusion-tensor MRI: theory, experimental design and data analysis - a technical review. NMR Biomed. 2002; 15(7-8):456-467. [PubMed: 12489095]

61. Mac Donald CL, Dikranian K, Song SK, et al. Detection of traumatic axonal injury with diffusion tensor imaging in a mouse model of traumatic brain injury. Exp Neurol. 2007; 205(1):116-131. [PubMed: 17368446]

62. Li J, Li XY, Feng DF, Gu L. Quantitative evaluation of microscopic injury with diffusion tensor imaging in a rat model of diffuse axonal injury. Eur J Neurosci. 2011; 33(5):933-945. [PubMed: 21385236] 
63. Rutgers DR, Fillard P, Paradot G, et al. Diffusion tensor imaging characteristics of the corpus callosum in mild, moderate, and severe traumatic brain injury. AJNR Am J Neuroradiol. 2008; 29(9):1730-1735. [PubMed: 18617586]

64. Inglese M, Makani S, Johnson G, et al. Diffuse axonal injury in mild traumatic brain injury: a diffusion tensor imaging study. J Neurosurg. 2005; 103(2):298-303. [PubMed: 16175860]

65. Huisman TA, Schwamm LH, Schaefer PW, et al. Diffusion tensor imaging as potential biomarker of white matter injury in diffuse axonal injury. AJNR Am J Neuroradiol. 2004; 25(3):370-376. [PubMed: 15037457]

66. Kumar R, Gupta RK, Husain M, et al. Comparative evaluation of corpus callosum DTI metrics in acute mild and moderate traumatic brain injury: its correlation with neuropsychometric tests. Brain Inj. 2009; 23(7):675-685. [PubMed: 19557571]

67. Lipton ML, Gellella E, Lo C, et al. Multifocal white matter ultrastructural abnormalities in mild traumatic brain injury with cognitive disability: a voxel-wise analysis of diffusion tensor imaging. J Neurotrauma. 2008; 25(11):1335-1342. [PubMed: 19061376]

68. Xu J, Rasmussen IA, Lagopoulos J, Haberg A. Diffuse axonal injury in severe traumatic brain injury visualized using high-resolution diffusion tensor imaging. J Neurotrauma. 2007; 24(5):753765. [PubMed: 17518531]

69. Grossman EJ, Ge Y, Jensen JH, et al. Thalamus and Cognitive Impairment in Mild Traumatic Brain Injury: A Diffusional Kurtosis Imaging Study. J Neurotrauma. 2011; 29(13):2318-2327. [PubMed: 21639753]

70. Lo C, Shifteh K, Gold T, Bello JA, Lipton ML. Diffusion tensor imaging abnormalities in patients with mild traumatic brain injury and neurocognitive impairment. Journal of computer assisted tomography. 2009; 33(2):293-297. [PubMed: 19346863]

71. Holli KK, Waljas M, Harrison L, et al. Mild traumatic brain injury: tissue texture analysis correlated to neuropsychological and DTI findings. Academic radiology. 2010; 17(9):1096-1102. [PubMed: 20605490]

72. Singh M, Jeong J, Hwang D, Sungkarat W, Gruen P. Novel diffusion tensor imaging methodology to detect and quantify injured regions and affected brain pathways in traumatic brain injury. Magn Reson Imaging. 2010; 28(1):22-40. [PubMed: 19608369]

73. Palacios EM, Fernandez-Espejo D, Junque C, et al. Diffusion tensor imaging differences relate to memory deficits in diffuse traumatic brain injury. BMC neurology. 2011; 11:24. [PubMed: 21345223]

74. Niogi SN, Mukherjee P, Ghajar J, et al. Structural dissociation of attentional control and memory in adults with and without mild traumatic brain injury. Brain. 2008; 131(12):3209-3221. [PubMed: 18952679]

75. Geary EK, Kraus MF, Pliskin NH, Little DM. Verbal learning differences in chronic mild traumatic brain injury. Journal of the International Neuropsychological Society. 2010; 16(3):506516. [PubMed: 20188015]

76. Mac Donald CL, Johnson AM, Cooper D, et al. Detection of blast-related traumatic brain injury in U.S. military personnel. N Engl J Med. 2011; 364(22):2091-2100. [PubMed: 21631321]

77. Newcombe VF, Williams GB, Scoffings D, et al. Aetiological differences in neuroanatomy of the vegetative state: insights from diffusion tensor imaging and functional implications. J Neurol Neurosurg Psychiatry. 2010; 81(5):552-561. [PubMed: 20460593]

78. Tollard E, Galanaud D, Perlbarg V, et al. Experience of diffusion tensor imaging and $1 \mathrm{H}$ spectroscopy for outcome prediction in severe traumatic brain injury: Preliminary results. Crit Care Med. 2009; 37(4):1448-1455. [PubMed: 19242330]

79. Miles L, Grossman RI, Johnson G, et al. Short-term DTI predictors of cognitive dysfunction in mild traumatic brain injury. Brain Inj. 2008; 22(2):115-122. [PubMed: 18240040]

80. Lipton ML, Gulko E, Zimmerman ME, et al. Diffusion-Tensor Imaging Implicates Prefrontal Axonal Injury in Executive Function Impairment Following Very Mild Traumatic Brain Injury. Radiology. 2009; 252(3):816-824. [PubMed: 19567646]

81. Arfanakis K, Haughton VM, Carew JD, et al. Diffusion tensor MR imaging in diffuse axonal injury. AJNR Am J Neuroradiol. 2002; 23(5):794-802. [PubMed: 12006280] 
82. Newcombe V, Chatfield D, Outtrim J, et al. Mapping traumatic axonal injury using diffusion tensor imaging: correlations with functional outcome. PLoS One. 2011; 6(5):e19214. [PubMed: 21573228]

83. Kumar R, Husain M, Gupta RK, et al. Serial changes in the white matter diffusion tensor imaging metrics in moderate traumatic brain injury and correlation with neuro-cognitive function. $\mathrm{J}$ Neurotrauma. 2009; 26(4):481-495. [PubMed: 19196176]

84. Sidaros A, Engberg AW, Sidaros K, et al. Diffusion tensor imaging during recovery from severe traumatic brain injury and relation to clinical outcome: a longitudinal study. Brain. 2008; 131(2): 559-572. [PubMed: 18083753]

85. Perlbarg V, Puybasset L, Tollard E, et al. Relation between brain lesion location and clinical outcome in patients with severe traumatic brain injury: a diffusion tensor imaging study using voxel-based approaches. Hum Brain Mapp. 2009; 30(12):3924-3933. [PubMed: 19507154]

86. Voss HU, Uluc AM, Dyke JP, et al. Possible axonal regrowth in late recovery from the minimally conscious state. J Clin Invest. 2006; 116(7):2005-2011. [PubMed: 16823492]

87. Rizzo, J. [Accessed July 26, 2012] Military surges MRIs to war zone to diagnose brain injuries. 2011. http://security.blogs.cnn.com/2011/11/01/military-surges-mris-to-war-zone-to-diagnosebrain-injuries/

88. Xydakis MS, Butman JA, Pierpaoli C. Blast-related traumatic brain injury in U.S. military personnel. N Engl J Med. 2011; 365(9):859. author reply 860-851. [PubMed: 21879909]

89. Salat D, Ward A, Kaye JA, Janowsky JS. Sex differences in the corpus callosum with aging. Neurobiology of aging. 1997; 18(2):191-197. [PubMed: 9258896]

90. Salat DH, Tuch DS, Greve DN, et al. Age-related alterations in white matter microstructure measured by diffusion tensor imaging. Neurobiology of aging. 2005; 26(8):1215-1227. [PubMed: 15917106]

91. Jones DK. The effect of gradient sampling schemes on measures derived from diffusion tensor MRI: a Monte Carlo study. Magn Reson Med. 2004; 51(4):807-815. [PubMed: 15065255]

92. Tensaouti F, Lahlou I, Clarisse P, Lotterie JA, Berry I. Quantitative and reproducibility study of four tractography algorithms used in clinical routine. J Magn Reson Imaging. 2011; 34(1):165172. [PubMed: 21618329]

93. Wang JY, Abdi H, Bakhadirov K, Diaz-Arrastia R, Devous MD Sr. A comprehensive reliability assessment of quantitative diffusion tensor tractography. Neuroimage. 2012; 60(2):1127-1138. [PubMed: 22227883]

94. Reese TG, Benner T, Wang R, Feinberg DA, Wedeen VJ. Halving imaging time of whole brain diffusion spectrum imaging and diffusion tractography using simultaneous image refocusing in EPI. J Magn Reson Imaging. 2009; 29(3):517-522. [PubMed: 19243032]

95. Setsompop K, Gagoski BA, Polimeni JR, et al. Blipped-controlled aliasing in parallel imaging for simultaneous multislice echo planar imaging with reduced g-factor penalty. Magn Reson Med. 2012; 67(5):1210-1224. [PubMed: 21858868]

96. Reese TG, Heid O, Weisskoff RM, Wedeen VJ. Reduction of eddy-current-induced distortion in diffusion MRI using a twice-refocused spin echo. Magn Reson Med. 2003; 49(1):177-182. [PubMed: 12509835]

97. Kraus MF, Susmaras T, Caughlin BP, et al. White matter integrity and cognition in chronic traumatic brain injury: a diffusion tensor imaging study. Brain. 2007; 130(10):2508-2519. [PubMed: 17872928]

98. Messe A, Caplain S, Paradot G, et al. Diffusion tensor imaging and white matter lesions at the subacute stage in mild traumatic brain injury with persistent neurobehavioral impairment. Hum Brain Mapp. 2011; 32(6):999-1011. [PubMed: 20669166]

99. Smith SM, Jenkinson M, Johansen-Berg H, et al. Tract-based spatial statistics: voxelwise analysis of multi-subject diffusion data. Neuroimage. 2006; 31(4):1487-1505. [PubMed: 16624579]

100. Smith SM, Johansen-Berg H, Jenkinson M, et al. Acquisition and voxelwise analysis of multisubject diffusion data with tract-based spatial statistics. Nat Protoc. 2007; 2(3):499-503. [PubMed: 17406613] 
101. Cubon VA, Putukian M, Boyer C, Dettwiler A. A diffusion tensor imaging study on the white matter skeleton in individuals with sports-related concussion. J Neurotrauma. 2011; 28(2):189201. [PubMed: 21083414]

102. Kinnunen KM, Greenwood R, Powell JH, et al. White matter damage and cognitive impairment after traumatic brain injury. Brain. 2011; 134(2):449-463. [PubMed: 21193486]

103. Wada T, Asano Y, Shinoda J. Decreased Fractional Anisotropy Evaluated Using Tract-Based Spatial Statistics and Correlated with Cognitive Dysfunction in Patients with Mild Traumatic Brain Injury in the Chronic Stage. AJNR Am J Neuroradiol. 201210.3174/ajnr.A3141

104. Palacios EM, Sala-Llonch R, Junque C, et al. White matter integrity related to functional working memory networks in traumatic brain injury. Neurology. 2012; 78(12):852-860. [PubMed: 22345222]

105. Palacios EM, Sala-Llonch R, Junque C, et al. Long-term declarative memory deficits in diffuse TBI: Correlations with cortical thickness, white matter integrity and hippocampal volume. Cortex. 201210.1016/j.cortex.201202011

106. Smith SM, Jenkinson M, Woolrich MW, et al. Advances in functional and structural MR image analysis and implementation as FSL. Neuroimage. 2004; 23 (Suppl 1):S208-219. [PubMed: 15501092]

107. Jenkinson M, Beckmann CF, Behrens TE, Woolrich MW, Smith SM. Fsl. Neuroimage. 2011; 62(2):782-90. [PubMed: 21979382]

108. Wang, R.; Wedeen, VJ.; Athinoula, A. Martinos Center for Biomedical Imaging. Massachusetts General Hospital; Boston, MA: 2010. http://www.trackvis.org [Accessed September 1, 2012]

109. Jiang H, van Zijl PC, Kim J, Pearlson GD, Mori S. DtiStudio: resource program for diffusion tensor computation and fiber bundle tracking. Computer methods and programs in biomedicine. 2006; 81(2):106-116. [PubMed: 16413083]

110. Pieper S, Halle M, Kikinis R. 3D Slicer. Proceedings of the 1st IEEE International Symposium on Biomedical Imaging: From Nano to Macro. 2004:632-635.

111. Talairach, J.; Tournoux, P. Co-Planar Stereotaxic Atlas of the Human Brain. New York: Thieme Medical Publishers; 1988.

112. Mazziotta J, Toga A, Evans A, et al. A probabilistic atlas and reference system for the human brain: International Consortium for Brain Mapping (ICBM). Philos Trans R Soc Lond B Biol Sci. 2001; 356(1412):1293-1322. [PubMed: 11545704]

113. McConnell Brain Imaging Centre, Montreal Neurological Institute. [Accessed September 1, 2012] 2012. www.bic.mni.mcgill.ca/ServicesAtlases/HomePage

114. Laboratory of NeuroImaging. University of California; Los Angeles: 2012. www.loni.ucla.edu/ Atlases [Accessed September 1, 2012]

115. Parvizi J, Damasio AR. Neuroanatomical correlates of brainstem coma. Brain. 2003; 126(Pt 7): 1524-1536. [PubMed: 12805123]

116. Wellcome Trust Centre for Neuroimaging. [Accessed September 1, 2012] Statistical Parametric Mapping. 2012. www.fil.ion.ucl.ac.uk/spm

117. McCausland Center for Brain Imaging. University of South Carolina; 2012. www.mccauslandcenter.sc.edu/mricro/mricron [Accessed September 1, 2012]

118. Reuter M, Schmansky NJ, Rosas HD, Fischl B. Within-subject template estimation for unbiased longitudinal image analysis. Neuroimage. 2012; 61(4):1402-1418. [PubMed: 22430496]

119. Oishi K, Faria A, Jiang H, et al. Atlas-based whole brain white matter analysis using large deformation diffeomorphic metric mapping: application to normal elderly and Alzheimer's disease participants. Neuroimage. 2009; 46(2):486-499. [PubMed: 19385016]

120. Janke AL, de Zubicaray G, Rose SE, et al. 4D deformation modeling of cortical disease progression in Alzheimer's dementia. Magn Reson Med. 2001; 46(4):661-666. [PubMed: 11590641]

121. Mori S, Crain BJ, Chacko VP, van Zijl PC. Three-dimensional tracking of axonal projections in the brain by magnetic resonance imaging. Ann Neurol. 1999; 45(2):265-269. [PubMed: 9989633] 
122. Jones DK, Simmons A, Williams SC, Horsfield MA. Non-invasive assessment of axonal fiber connectivity in the human brain via diffusion tensor MRI. Magn Reson Med. 1999; 42(1):37-41. [PubMed: 10398948]

123. Basser PJ, Pajevic S, Pierpaoli C, Duda J, Aldroubi A. In vivo fiber tractography using DT-MRI data. Magn Reson Med. 2000; 44(4):625-632. [PubMed: 11025519]

124. Catani M, Thiebaut de Schotten M. A diffusion tensor imaging tractography atlas for virtual in vivo dissections. Cortex. 2008; 44(8):1105-1132. [PubMed: 18619589]

125. Hansen B, Flint JJ, Heon-Lee C, et al. Diffusion tensor microscopy in human nervous tissue with quantitative correlation based on direct histological comparison. Neuroimage. 2011; 57(4):14581465. [PubMed: 21575730]

126. Wang JY, Bakhadirov K, Devous MD Sr, et al. Diffusion tensor tractography of traumatic diffuse axonal injury. Arch Neurol. 2008; 65(5):619-626. [PubMed: 18474737]

127. Wang JY, Bakhadirov K, Abdi H, et al. Longitudinal changes of structural connectivity in traumatic axonal injury. Neurology. 2011; 77(9):818-826. [PubMed: 21813787]

128. Warner MA, Marquez de la Plata C, Spence J, et al. Assessing spatial relationships between axonal integrity, regional brain volumes, and neuropsychological outcomes after traumatic axonal injury. J Neurotrauma. 2010; 27(12):2121-2130. [PubMed: 20874032]

129. Maxwell WL, MacKinnon MA, Stewart JE, Graham DI. Stereology of cerebral cortex after traumatic brain injury matched to the Glasgow outcome score. Brain. 2010; 133(Pt 1):139-160. [PubMed: 19897544]

130. Gennarelli TA, Thibault LE, Adams JH, et al. Diffuse axonal injury and traumatic coma in the primate. Ann Neurol. 1982; 12(6):564-574. [PubMed: 7159060]

131. Smith DH, Nonaka M, Miller R, et al. Immediate coma following inertial brain injury dependent on axonal damage in the brainstem. J Neurosurg. 2000; 93(2):315-322. [PubMed: 10930019]

132. Wilde EA, McCauley SR, Hunter JV, et al. Diffusion tensor imaging of acute mild traumatic brain injury in adolescents. Neurology. 2008; 70(12):948-955. [PubMed: 18347317]

133. Mulkern RV, Haker SJ, Maier SE. On high b diffusion imaging in the human brain: ruminations and experimental insights. Magn Reson Imaging. 2009; 27(8):1151-1162. [PubMed: 19520535]

134. Yoshiura T, Wu O, Zaheer A, Reese TG, Sorensen AG. Highly diffusion-sensitized MRI of brain: dissociation of gray and white matter. Magn Reson Med. 2001; 45(5):734-740. [PubMed: 11323798]

135. Melhem ER, Itoh R, Jones L, Barker PB. Diffusion tensor MR imaging of the brain: effect of diffusion weighting on trace and anisotropy measurements. AJNR Am J Neuroradiol. 2000; 21(10):1813-1820. [PubMed: 11110532]

136. Pasternak O, Sochen N, Gur Y, Intrator N, Assaf Y. Free water elimination and mapping from diffusion MRI. Magn Reson Med. 2009; 62(3):717-730. [PubMed: 19623619]

137. Bazarian JJ, Zhong J, Blyth B, et al. Diffusion tensor imaging detects clinically important axonal damage after mild traumatic brain injury: a pilot study. J Neurotrauma. 2007; 24(9):1447-1459. [PubMed: 17892407]

138. Wedeen VJ, Rosene DL, Wang R, et al. The geometric structure of the brain fiber pathways. Science. 2012; 335(6076):1628-1634. [PubMed: 22461612]

139. Wedeen VJ, Hagmann P, Tseng WY, Reese TG, Weisskoff RM. Mapping complex tissue architecture with diffusion spectrum magnetic resonance imaging. Magn Reson Med. 2005; 54(6):1377-1386. [PubMed: 16247738]

140. Tuch DS, Reese TG, Wiegell MR, Wedeen VJ. Diffusion MRI of complex neural architecture. Neuron. 2003; 40(5):885-895. [PubMed: 14659088]

141. Frank LR. Anisotropy in high angular resolution diffusion-weighted MRI. Magn Reson Med. 2001; 45(6):935-939. [PubMed: 11378869]

142. Clark CA, Le Bihan D. Water diffusion compartmentation and anisotropy at high $b$ values in the human brain. Magn Reson Med. 2000; 44(6):852-859. [PubMed: 11108621]

143. Jansons KM, Alexander DC. Persistent Angular Structure: new insights from diffusion MRI data. Dummy version Information processing in medical imaging: proceedings of the conference. $2003 ; 18: 672-683$. 
144. Tournier JD, Calamante F, Gadian DG, Connelly A. Direct estimation of the fiber orientation density function from diffusion-weighted MRI data using spherical deconvolution. Neuroimage. 2004; 23(3):1176-1185. [PubMed: 15528117]

145. Assaf Y, Freidlin RZ, Rohde GK, Basser PJ. New modeling and experimental framework to characterize hindered and restricted water diffusion in brain white matter. Magn Reson Med. 2004; 52(5):965-978. [PubMed: 15508168]

146. Leergaard TB, White NS, de Crespigny A, et al. Quantitative histological validation of diffusion MRI fiber orientation distributions in the rat brain. PLoS One. 2010; 5(1):e8595. [PubMed: 20062822]

147. Schmahmann JD, Pandya DN, Wang R, et al. Association fibre pathways of the brain: parallel observations from diffusion spectrum imaging and autoradiography. Brain. 2007; 130(3):630653. [PubMed: 17293361]

148. Tournier, JD. The Biophysics of Crossing Fibers. In: Jones, DK., editor. Diffusion MRI. Oxford: Oxford University Press; 2011. p. 465-481.

149. Alexander, DC.; Seunarine, KK. Mathematics of Crossing Fibers. In: Jones, DK., editor. Diffusion MRI. Oxford: Oxford University Press; 2011. p. 451-464.

150. Hess CP, Mukherjee P. Visualizing white matter pathways in the living human brain: diffusion tensor imaging and beyond. Neuroimaging Clin N Am. 2007; 17(4):407-426. [PubMed: 17983960]

151. Shin SS, Verstynen T, Pathak S, et al. High-definition fiber tracking for assessment of neurological deficit in a case of traumatic brain injury: finding, visualizing, and interpreting small sites of damage. J Neurosurg. 2012; 116(5):1062-9. [PubMed: 22381003]

152. Edlow BL, Takahashi E, Wu O, et al. Neuroanatomic Connectivity of the Human Ascending Arousal System Critical to Consciousness and Its Disorders. J Neuropathol Exp Neurol. 2012; 71(6):531-546. [PubMed: 22592840]

153. Behrens TE, Woolrich MW, Jenkinson M, et al. Characterization and propagation of uncertainty in diffusion-weighted MR imaging. Magn Reson Med. 2003; 50(5):1077-1088. [PubMed: 14587019]

154. Behrens TE, Johansen-Berg H, Woolrich MW, et al. Non-invasive mapping of connections between human thalamus and cortex using diffusion imaging. Nat Neurosci. 2003; 6(7):750-757. [PubMed: 12808459]

155. Raichle ME, MacLeod AM, Snyder AZ, et al. A default mode of brain function. Proc Natl Acad Sci U S A. 2001; 98(2):676-682. [PubMed: 11209064]

156. Raichle ME, Snyder AZ. A default mode of brain function: a brief history of an evolving idea. Neuroimage. 2007; 37(4):1083-1090. [PubMed: 17719799]

157. Shulman GL, Fiez JA, Corbetta M, et al. Common blood flow changes across visual tasks: II. Decreases in cerebral cortex. J Cogn Neurosci. 1997; 9:648-663. [PubMed: 23965122]

158. Binder JR, Frost JA, Hammeke TA, et al. Conceptual processing during the conscious resting state. A functional MRI study. J Cogn Neurosci. 1999; 11(1):80-95. [PubMed: 9950716]

159. Seeley WW, Menon V, Schatzberg AF, et al. Dissociable intrinsic connectivity networks for salience processing and executive control. J Neurosci. 2007; 27(9):2349-2356. [PubMed: 17329432]

160. Tang L, Ge Y, Sodickson DK, et al. Thalamic resting-state functional networks: disruption in patients with mild traumatic brain injury. Radiology. 2011; 260(3):831-840. [PubMed: 21775670]

161. Fox MD, Snyder AZ, Vincent JL, et al. The human brain is intrinsically organized into dynamic, anticorrelated functional networks. Proc Natl Acad Sci U S A. 2005; 102(27):9673-9678. [PubMed: 15976020]

162. Vincent JL, Snyder AZ, Fox MD, et al. Coherent spontaneous activity identifies a hippocampalparietal memory network. Journal of neurophysiology. 2006; 96(6):3517-3531. [PubMed: 16899645]

163. Biswal B, Yetkin FZ, Haughton VM, Hyde JS. Functional connectivity in the motor cortex of resting human brain using echo-planar MRI. Magn Reson Med. 1995; 34(4):537-541. [PubMed: 8524021] 
164. Buckner RL, Andrews-Hanna JR, Schacter DL. The brain's default network: anatomy, function, and relevance to disease. Ann N Y Acad Sci. 2008; 1124:1-38. [PubMed: 18400922]

165. Fransson P, Marrelec G. The precuneus/posterior cingulate cortex plays a pivotal role in the default mode network: Evidence from a partial correlation network analysis. Neuroimage. 2008; 42(3):1178-1184. [PubMed: 18598773]

166. Fox MD, Greicius M. Clinical applications of resting state functional connectivity. Frontiers in systems neuroscience. 2010; 4:19. [PubMed: 20592951]

167. Beckmann CF, Smith SM. Probabilistic independent component analysis for functional magnetic resonance imaging. IEEE Trans Med Imaging. 2004; 23(2):137-152. [PubMed: 14964560]

168. Boly M, Phillips C, Tshibanda L, et al. Intrinsic brain activity in altered states of consciousness: how conscious is the default mode of brain function? Ann N Y Acad Sci. 2008; 1129:119-129. [PubMed: 18591474]

169. Johnson B, Zhang K, Gay M, et al. Alteration of brain default network in subacute phase of injury in concussed individuals: resting-state fMRI study. Neuroimage. 2012; 59(1):511-518. [PubMed: 21846504]

170. Zhang K, Johnson B, Gay M, et al. Default mode network in concussed individuals in response to the YMCA physical stress test. J Neurotrauma. 2012; 29(5):756-765. [PubMed: 22040294]

171. Sharp DJ, Beckmann CF, Greenwood R, et al. Default mode network functional and structural connectivity after traumatic brain injury. Brain. 2011; 134(8):2233-2247. [PubMed: 21841202]

172. Hillary FG, Slocomb J, Hills EC, et al. Changes in resting connectivity during recovery from severe traumatic brain injury. International journal of psychophysiology: official journal of the International Organization of Psychophysiology. 2011; 82(1):115-123. [PubMed: 21473890]

173. Bonnelle V, Leech R, Kinnunen KM, et al. Default mode network connectivity predicts sustained attention deficits after traumatic brain injury. J Neurosci. 2011; 31(38):13442-13451. [PubMed: 21940437]

174. Mayer AR, Mannell MV, Ling J, Gasparovic C, Yeo RA. Functional connectivity in mild traumatic brain injury. Hum Brain Mapp. 2011; 32(11):1825-1835. [PubMed: 21259381]

175. Bonnelle V, Ham TE, Leech R, et al. Salience network integrity predicts default mode network function after traumatic brain injury. Proc Natl Acad Sci U S A. 2012; 109(12):4690-4695. [PubMed: 22393019]

176. Stevens MC, Lovejoy D, Kim J, et al. Multiple resting state network functional connectivity abnormalities in mild traumatic brain injury. Brain imaging and behavior. 2012; 6(2):293-318. [PubMed: 22555821]

177. Singh KD, Fawcett IP. Transient and linearly graded deactivation of the human default-mode network by a visual detection task. Neuroimage. 2008; 41(1):100-112. [PubMed: 18375149]

178. Vanhaudenhuyse A, Demertzi A, Schabus M, et al. Two distinct neuronal networks mediate the awareness of environment and of self. J Cogn Neurosci. 2011; 23(3):570-578. [PubMed: 20515407]

179. Boly M, Balteau E, Schnakers C, et al. Baseline brain activity fluctuations predict somatosensory perception in humans. Proc Natl Acad Sci U S A. 2007; 104(29):12187-12192. [PubMed: 17616583]

180. Thibaut A, Bruno MA, Chatelle C, et al. Metabolic activity in external and internal awareness networks in severely brain-damaged patients. Journal of rehabilitation medicine: official journal of the UEMS European Board of Physical and Rehabilitation Medicine. 2012; 44(5):487-494. [PubMed: 22366927]

181. Biswal BB, Mennes M, Zuo XN, et al. Toward discovery science of human brain function. Proc Natl Acad Sci U S A. 2010; 107(10):4734-4739. [PubMed: 20176931]

182. Greicius MD, Kiviniemi V, Tervonen O, et al. Persistent default-mode network connectivity during light sedation. Hum Brain Mapp. 2008; 29(7):839-847. [PubMed: 18219620]

183. Fukunaga M, Horovitz SG, van Gelderen P, et al. Large-amplitude, spatially correlated fluctuations in BOLD fMRI signals during extended rest and early sleep stages. Magn Reson Imaging. 2006; 24(8):979-992. [PubMed: 16997067] 
184. Boveroux P, Vanhaudenhuyse A, Bruno MA, et al. Breakdown of within- and between-network resting state functional magnetic resonance imaging connectivity during propofol-induced loss of consciousness. Anesthesiology. 2010; 113(5):1038-1053. [PubMed: 20885292]

185. Yan C, Liu D, He Y, et al. Spontaneous brain activity in the default mode network is sensitive to different resting-state conditions with limited cognitive load. PLoS One. 2009; 4(5):e5743. [PubMed: 19492040]

186. Jo HJ, Saad ZS, Simmons WK, Milbury LA, Cox RW. Mapping sources of correlation in resting state FMRI, with artifact detection and removal. Neuroimage. 2010; 52(2):571-582. [PubMed: 20420926]

187. Kwong KK, Belliveau JW, Chesler DA, et al. Dynamic magnetic resonance imaging of human brain activity during primary sensory stimulation. Proc Natl Acad Sci U S A. 1992; 89(12):56755679. [PubMed: 1608978]

188. Ogawa S, Tank DW, Menon R, et al. Intrinsic signal changes accompanying sensory stimulation: functional brain mapping with magnetic resonance imaging. Proc Natl Acad Sci U S A. 1992; 89(13):5951-5955. [PubMed: 1631079]

189. Smith K. Brain imaging: fMRI 2. 0. Nature. 2012; 484(7392):24-26. [PubMed: 22481337]

190. Kasahara M, Menon DK, Salmond CH, et al. Traumatic brain injury alters the functional brain network mediating working memory. Brain Inj. 2011; 25(12):1170-1187. [PubMed: 21932994]

191. Kasahara M, Menon DK, Salmond CH, et al. Altered functional connectivity in the motor network after traumatic brain injury. Neurology. 2010; 75(2):168-176. [PubMed: 20625170]

192. Mayer AR, Yang Z, Yeo RA, et al. A functional MRI study of multimodal selective attention following mild traumatic brain injury. Brain imaging and behavior. 2012; 6(2):343-354. [PubMed: 22673802]

193. Newsome MR, Scheibel RS, Hanten G, et al. Brain activation while thinking about the self from another person's perspective after traumatic brain injury in adolescents. Neuropsychology. 2010; 24(2):139-147. [PubMed: 20230107]

194. Detre JA, Leigh JS, Williams DS, Koretsky AP. Perfusion imaging. Magn Reson Med. 1992; 23(1):37-45. [PubMed: 1734182]

195. Fernandez-Seara MA, Edlow BL, Hoang A, et al. Minimizing acquisition time of arterial spin labeling at 3T. Magn Reson Med. 2008; 59(6):1467-1471. [PubMed: 18506806]

196. Chen Y, Wang DJ, Detre JA. Test-retest reliability of arterial spin labeling with common labeling strategies. J Magn Reson Imaging. 2011; 33(4):940-949. [PubMed: 21448961]

197. Kim J, Whyte J, Patel S, et al. Methylphenidate modulates sustained attention and cortical activation in survivors of traumatic brain injury: a perfusion fMRI study. Psychopharmacology. 2012; 222(1):47-57. [PubMed: 22203319]

198. Helle M, Norris DG, Rufer S, et al. Superselective pseudocontinuous arterial spin labeling. Magn Reson Med. 2010; 64(3):777-786. [PubMed: 20597127]

199. Detre JA, Wang J. Technical aspects and utility of fMRI using BOLD and ASL. Clin Neurophysiol. 2002; 113(5):621-634. [PubMed: 11976042]

200. Kim J, Whyte J, Patel S, et al. Resting cerebral blood flow alterations in chronic traumatic brain injury: an arterial spin labeling perfusion FMRI study. J Neurotrauma. 2010; 27(8):1399-1411. [PubMed: 20528163]

201. Kim J, Whyte J, Patel S, et al. A Perfusion fMRI Study of the Neural Correlates of SustainedAttention and Working-Memory Deficits in Chronic Traumatic Brain Injury. Neurorehabil Neural Repair. 2012; 26(7):870-880. [PubMed: 22357634]

202. Ge Y, Patel MB, Chen Q, et al. Assessment of thalamic perfusion in patients with mild traumatic brain injury by true FISP arterial spin labelling MR imaging at 3T. Brain Inj. 2009; 23(7):666674. [PubMed: 19557570]

203. Dai W, Garcia D, de Bazelaire C, Alsop DC. Continuous flow-driven inversion for arterial spin labeling using pulsed radio frequency and gradient fields. Magn Reson Med. 2008; 60(6):14881497. [PubMed: 19025913]

204. Wu WC, Fernandez-Seara M, Detre JA, Wehrli FW, Wang J. A theoretical and experimental investigation of the tagging efficiency of pseudocontinuous arterial spin labeling. Magn Reson Med. 2007; 58(5):1020-1027. [PubMed: 17969096] 
205. Wong EC, Cronin M, Wu WC, et al. Velocity-selective arterial spin labeling. Magn Reson Med. 2006; 55(6):1334-1341. [PubMed: 16700025]

206. Wu WC, Edlow BL, Elliot MA, Wang J, Detre JA. Physiological modulations in arterial spin labeling perfusion magnetic resonance imaging. IEEE Trans Med Imaging. 2009; 28(5):703-709. [PubMed: 19150788]

207. Zaharchuk G. Theoretical basis of hemodynamic MR imaging techniques to measure cerebral blood volume, cerebral blood flow, and permeability. AJNR Am J Neuroradiol. 2007; 28(10): 1850-1858. [PubMed: 17998415]

208. Reivich M, Kuhl D, Wolf A, et al. The [18F]fluorodeoxyglucose method for the measurement of local cerebral glucose utilization in man. Circulation research. 1979; 44(1):127-137. [PubMed: 363301]

209. Greenberg JH, Reivich M, Alavi A, et al. Metabolic mapping of functional activity in human subjects with the [18F]fluorodeoxyglucose technique. Science. 1981; 212(4495):678-680. [PubMed: 6971492]

210. Laureys S, Goldman S, Phillips C, et al. Impaired effective cortical connectivity in vegetative state: preliminary investigation using PET. Neuroimage. 1999; 9(4):377-382. [PubMed: 10191166]

211. Bruno MA, Majerus S, Boly M, et al. Functional neuroanatomy underlying the clinical subcategorization of minimally conscious state patients. Journal of neurology. 2012; 259(6): 1087-1098. [PubMed: 22081100]

212. Schiff ND, Ribary U, Moreno DR, et al. Residual cerebral activity and behavioural fragments can remain in the persistently vegetative brain. Brain. 2002; 125(6):1210-1234. [PubMed: 12023311]

213. Boly M, Faymonville ME, Peigneux P, et al. Auditory processing in severely brain injured patients: differences between the minimally conscious state and the persistent vegetative state. Arch Neurol. 2004; 61(2):233-238. [PubMed: 14967772]

214. Xu V, Chan H, Lin AP, et al. MR spectroscopy in diagnosis and neurological decision-making. Semin Neurol. 2008; 28(4):407-422. [PubMed: 18843570]

215. Ling G, Bandak F, Armonda R, Grant G, Ecklund J. Explosive blast neurotrauma. J Neurotrauma. 2009; 26(6):815-825. [PubMed: 19397423]

216. Bell RS, Vo AH, Neal CJ, et al. Military traumatic brain and spinal column injury: a 5-year study of the impact blast and other military grade weaponry on the central nervous system. J Trauma. 2009; 66(4 Suppl):S104-111. [PubMed: 19359953]

217. Warden D. Military TBI during the Iraq and Afghanistan wars. J Head Trauma Rehabil. 2006; 21(5):398-402. [PubMed: 16983225]

218. Vanderploeg RD, Schwab K, Walker WC, et al. Rehabilitation of traumatic brain injury in active duty military personnel and veterans: Defense and Veterans Brain Injury Center randomized controlled trial of two rehabilitation approaches. Arch Phys Med Rehabil. 2008; 89(12):22272238. [PubMed: 19061734]

219. Zoroya, G. For troops with brain trauma, a long journey back. USA Today; Tampa: Jul 29. 2010

220. Nakagawa A, Manley GT, Gean AD, et al. Mechanisms of primary blast-induced traumatic brain injury: insights from shock-wave research. J Neurotrauma. 2011; 28(6):1101-1119. [PubMed: 21332411]

221. Ropper A. Brain injuries from blasts. N Engl J Med. 2011; 364(22):2156-2157. [PubMed: 21631329]

222. Armonda RA, Bell RS, Vo AH, et al. Wartime traumatic cerebral vasospasm: recent review of combat casualties. Neurosurgery. 2006; 59(6):1215-1225. [PubMed: 17277684]

223. Bazarian JJ, Donnelly K, Peterson DR, et al. The Relation Between Posttraumatic Stress Disorder and Mild Traumatic Brain Injury Acquired During Operations Enduring Freedom and Iraqi Freedom: A Diffusion Tensor Imaging Study. J Head Trauma Rehabil. 201210.1097/HTR. $0 \mathrm{~b} 013 \mathrm{e} 318256 \mathrm{~d} 3 \mathrm{~d} 3$

224. Levin HS, Wilde E, Troyanskaya M, et al. Diffusion tensor imaging of mild to moderate blastrelated traumatic brain injury and its sequelae. J Neurotrauma. 2010; 27(4):683-694. [PubMed: 20088647] 
225. Hoge CW, McGurk D, Thomas JL, et al. Mild traumatic brain injury in U.S. Soldiers returning from Iraq. N Engl J Med. 2008; 358(5):453-463. [PubMed: 18234750]

226. McKee AC, Cantu RC, Nowinski CJ, et al. Chronic traumatic encephalopathy in athletes: progressive tauopathy after repetitive head injury. J Neuropathol Exp Neurol. 2009; 68(7):709_ 735. [PubMed: 19535999]

227. Slobounov S, Gay M, Johnson B, Zhang K. Concussion in athletics: ongoing clinical and brain imaging research controversies. Brain imaging and behavior. 2012; 6(2):224-243. [PubMed: 22669496]

228. Practice parameter: the management of concussion in sports (summary statement). Report of the Quality Standards Subcommittee. Neurology. 1997; 48(3):581-585. [PubMed: 9065530]

229. Alexander MP. Mild traumatic brain injury: pathophysiology, natural history, and clinical management. Neurology. 1995; 45(7):1253-1260. [PubMed: 7617178]

230. Zhang K, Johnson B, Pennell D, et al. Are functional deficits in concussed individuals consistent with white matter structural alterations: combined FMRI \& DTI study. Experimental brain research Experimentelle Hirnforschung Experimentation cerebrale. 2010; 204(1):57-70. [PubMed: 20496060]

231. Gandy S, Dekosky ST. APOE epsilon4 status and traumatic brain injury on the gridiron or the battlefield. Science translational medicine. 2012; 4(134):134ed134.

232. Andrews K, Murphy L, Munday R, Littlewood C. Misdiagnosis of the vegetative state: retrospective study in a rehabilitation unit. BMJ. 1996; 313(7048):13-16. [PubMed: 8664760]

233. Childs NL, Mercer WN, Childs HW. Accuracy of diagnosis of persistent vegetative state. Neurology. 1993; 43(8):1465-1467. [PubMed: 8350997]

234. Schnakers C, Vanhaudenhuyse A, Giacino J, et al. Diagnostic accuracy of the vegetative and minimally conscious state: clinical consensus versus standardized neurobehavioral assessment. BMC neurology. 2009; 9:35. [PubMed: 19622138]

235. Giacino JT, Kalmar K, Whyte J. The JFK Coma Recovery Scale-Revised: measurement characteristics and diagnostic utility. Arch Phys Med Rehabil. 2004; 85(12):2020-2029. [PubMed: 15605342]

236. Giacino JT, Whyte J, Bagiella E, et al. Placebo-controlled trial of amantadine for severe traumatic brain injury. N Engl J Med. 2012; 366(9):819-826. [PubMed: 22375973]

237. Whyte J, Myers R. Incidence of clinically significant responses to zolpidem among patients with disorders of consciousness: a preliminary placebo controlled trial. Am J Phys Med Rehabil. 2009; 88(5):410-418. [PubMed: 19620954]

238. Schiff ND, Giacino JT, Kalmar K, et al. Behavioural improvements with thalamic stimulation after severe traumatic brain injury. Nature. 2007; 448(7153):600-603. [PubMed: 17671503]

239. Fins JJ. The ethics of measuring and modulating consciousness: the imperative of minding time. Prog Brain Res. 2009; 177:371-382. [PubMed: 19818915]

240. Owen AM, Coleman MR, Boly M, et al. Detecting awareness in the vegetative state. Science. 2006; 313(5792):1402. [PubMed: 16959998]

241. Monti MM, Vanhaudenhuyse A, Coleman MR, et al. Willful modulation of brain activity in disorders of consciousness. N Engl J Med. 2010; 362(7):579-589. [PubMed: 20130250]

242. Ropper AH. Cogito ergo sum by MRI. N Engl J Med. 2010; 362(7):648-649. [PubMed: 20130248]

243. Coleman MR, Rodd JM, Davis MH, et al. Do vegetative patients retain aspects of language comprehension? Evidence from fMRI Brain. 2007; 130(10):2494-2507.

244. Coleman MR, Davis MH, Rodd JM, et al. Towards the routine use of brain imaging to aid the clinical diagnosis of disorders of consciousness. Brain. 2009; 132(9):2541-2552. [PubMed: 19710182]

245. Schiff ND, Rodriguez-Moreno D, Kamal A, et al. fMRI reveals large-scale network activation in minimally conscious patients. Neurology. 2005; 64(3):514-523. [PubMed: 15699384]

246. Fernandez-Espejo D, Junque C, Vendrell P, et al. Cerebral response to speech in vegetative and minimally conscious states after traumatic brain injury. Brain Inj. 2008; 22(11):882-890. [PubMed: 18850346] 
247. Laureys S, Lemaire C, Maquet P, Phillips C, Franck G. Cerebral metabolism during vegetative state and after recovery to consciousness. J Neurol Neurosurg Psychiatry. 1999; 67(1):121. [PubMed: 10454871]

248. Vanhaudenhuyse A, Noirhomme Q, Tshibanda LJ, et al. Default network connectivity reflects the level of consciousness in non-communicative brain-damaged patients. Brain. 2010; 133(1):161171. [PubMed: 20034928]

249. Silva S, Alacoque X, Fourcade O, et al. Wakefulness and loss of awareness: brain and brainstem interaction in the vegetative state. Neurology. 2010; 74(4):313-320. [PubMed: 20101037]

250. Liu AA, Voss HU, Dyke JP, Heier LA, Schiff ND. Arterial spin labeling and altered cerebral blood flow patterns in the minimally conscious state. Neurology. 2011; 77(16):1518-1523. [PubMed: 21940616]

251. Laureys S, Schiff ND. Coma and consciousness: paradigms (re)framed by neuroimaging. Neuroimage. 2012; 61(2):478-491. [PubMed: 22227888]

252. Bardin JC, Schiff ND, Voss HU. Pattern classification of volitional functional magnetic resonance imaging responses in patients with severe brain injury. Arch Neurol. 2012; 69(2):176181. [PubMed: 22332186]

253. Bardin JC, Fins JJ, Katz DI, et al. Dissociations between behavioural and functional magnetic resonance imaging-based evaluations of cognitive function after brain injury. Brain. 2011; 134(3):769-782. [PubMed: 21354974]

254. Moruzzi G, Magoun HW. Brain stem reticular formation and activation of the EEG. Electroencephalogr Clin Neurophysiol. 1949; 1(4):455-473. [PubMed: 18421835]

255. Steriade M. Arousal: revisiting the reticular activating system. Science. 1996; 272(5259):225226. [PubMed: 8602506]

256. Kinomura S, Larsson J, Gulyas B, Roland PE. Activation by attention of the human reticular formation and thalamic intralaminar nuclei. Science. 1996; 271(5248):512-515. [PubMed: 8560267]

257. Krzywinski M, Schein J, Birol I, et al. Circos: an information aesthetic for comparative genomics. Genome Res. 2009; 19(9):1639-1645. [PubMed: 19541911]

258. Sporns, O. Networks of the brain. Cambridge: MIT Press; 2011.

259. Sporns O, Tononi G, Kotter R. The human connectome: A structural description of the human brain. PLoS Comput Biol. 2005; 1(4):e42. [PubMed: 16201007]

260. Hagmann, P. From diffusion MRI to brain connectomics. Programme Doctoral en Informatique et Communications. Lausanne, EPFL: Université de Lausanne; 2005. p. 1-122.

261. Irimia A, Chambers MC, Torgerson CM, Van Horn JD. Circular representation of human cortical networks for subject and population-level connectomic visualization. Neuroimage. 2012; 60(2): 1340-1351. [PubMed: 22305988]

262. Irimia A, Chambers MC, Torgerson CM, et al. Patient-tailored connectomics visualization for the assessment of white matter atrophy in traumatic brain injury. Front Neurol. 2012; 3:10. [PubMed: 22363313]

263. Edlow BL, Diamond EL. Teaching NeuroImages: restricted diffusion in the corpus callosum after traumatic diffuse axonal injury. Neurology. 2010; 75(17):e69. [PubMed: 20975045]

264. Catani M, Jones DK, ffytche DH. Perisylvian language networks of the human brain. Ann Neurol. 2005; 57(1):8-16. [PubMed: 15597383]

265. Worsley, K. Statistical analysis of activation images. In: Jezzard, P.; Matthews, PM.; Smith, SM., editors. Functional MRI: An introduction to methods. Oxford: Oxford University Press; 2001.

266. Van Dijk KR, Hedden T, Venkataraman A, et al. Intrinsic functional connectivity as a tool for human connectomics: theory, properties, and optimization. Journal of neurophysiology. 2010; 103(1):297-321. [PubMed: 19889849] 

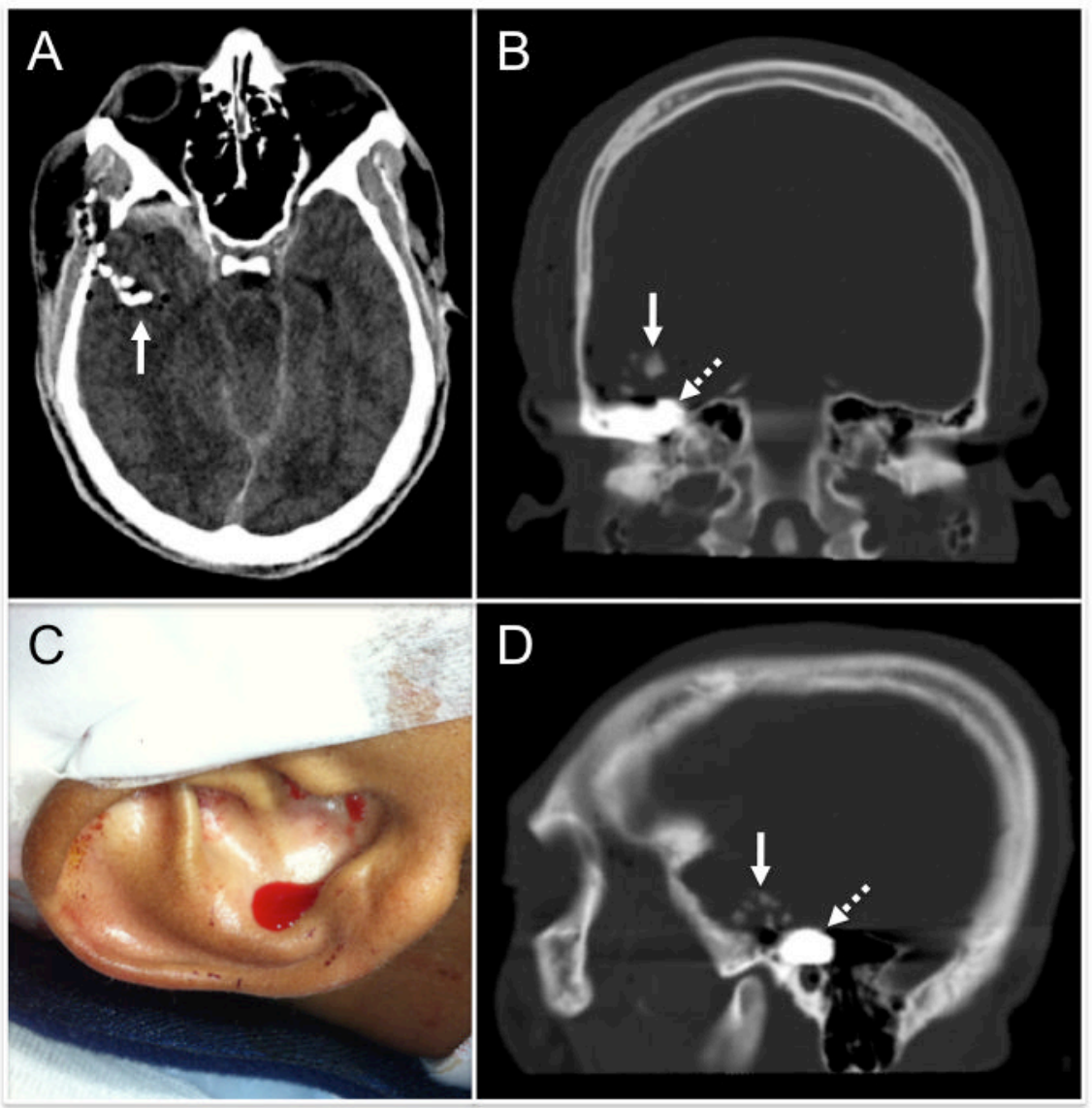

Figure 1.

Head CT of penetrating TBI in a 22-year-old man with a right temporal gunshot wound. (A) Axial CT demonstrates bone fragments in the right temporal lobe (arrow), subdural hemorrhage and pneumocephalus anterior to the right temporal pole, and compression of the basal cisterns. Coronal (B) and sagittal (D) bone-windowed CT shows the bone fragments (solid arrows) and a focal hyperdensity, representing the bullet (dashed arrows), which is lodged in the temporal bone at the level of the middle ear canal adjacent to the mastoid air cells. The right temporal bone fracture explains the presence of bloody cerebrospinal fluid leaking out of the patient's right ear $(\mathrm{C})$. 


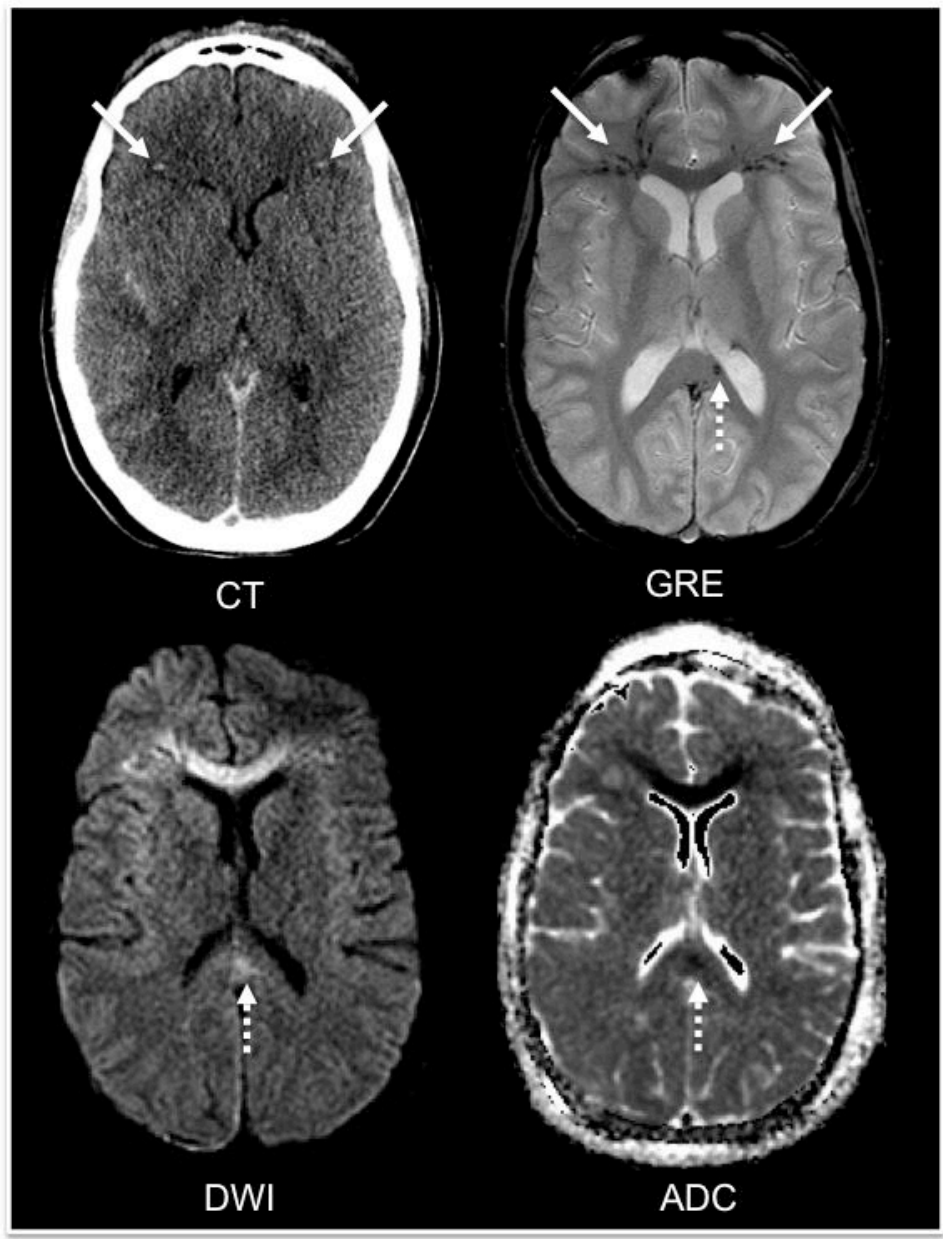

Figure 2.

Comparison of CT, GRE, DWI, and ADC detection of traumatic axonal injury in a 19-yearold female pedestrian hit by a car. After a 5-minute loss of consciousness, the patient was inattentive and somnolent on arrival to the Emergency Department. CT was performed on the day of the injury, and MRI was performed seven days later. Only two traumatic microbleeds (TMBs) are seen on CT, whereas multiple TMBs are seen on GRE in the bifrontal white matter (solid arrows). Restricted diffusion is present throughout the genu of the corpus callosum, as indicated by hyperintense signal on DWI and hypointense signal on ADC. In addition, a smaller focus of diffusion restriction associated with a TMB is seen in the splenium of the corpus callosum (dashed arrows). Adapted with permission from Edlow and Diamond, Neurology 2010. ${ }^{263}$ 


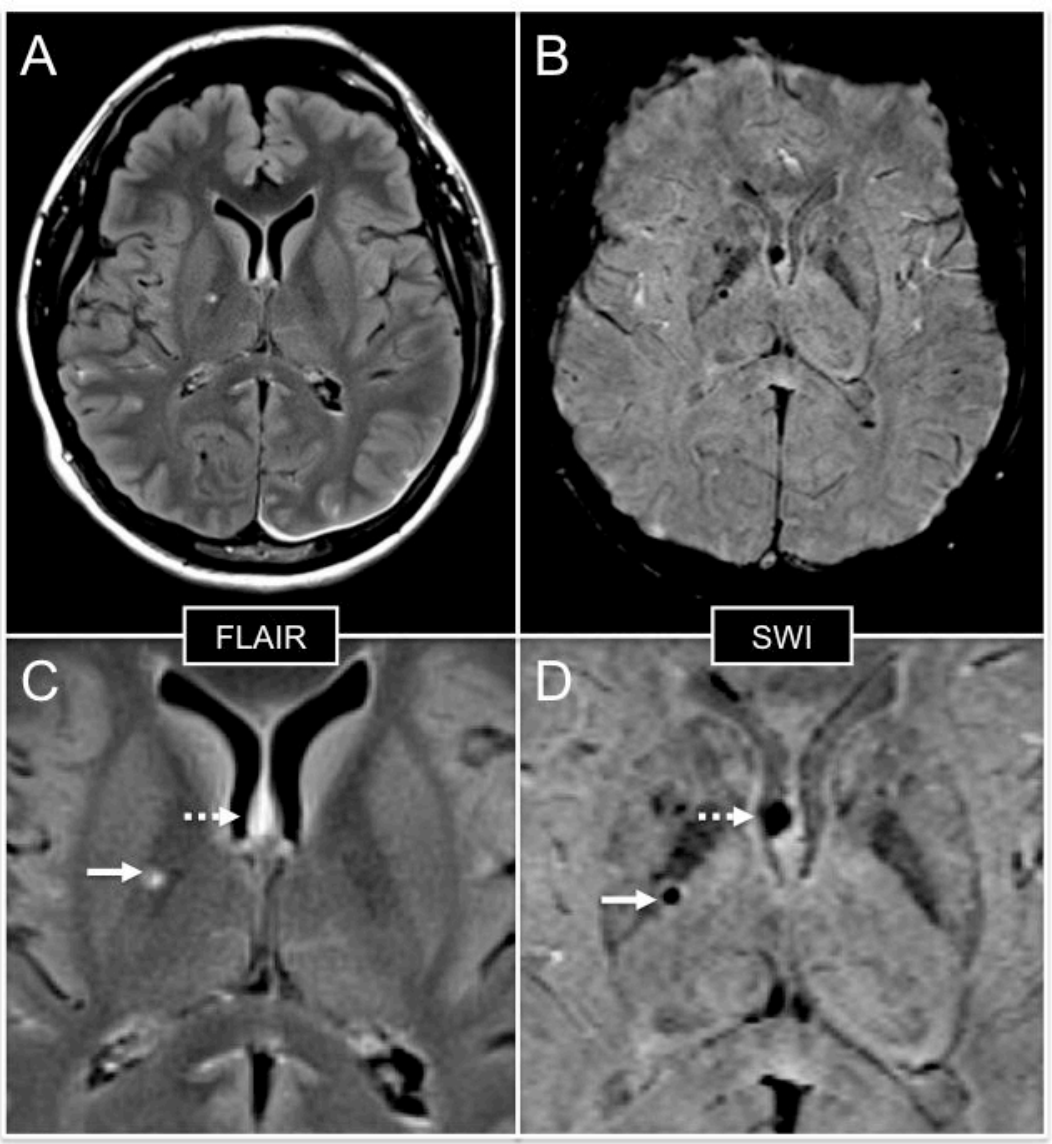

Figure 3.

T2-FLAIR and SWI data from a 27-year-old man hit by a car while riding his bike. The patient's admission Glasgow Coma Scale score was 7T (E1, M5, V1T), consistent with severe traumatic brain injury. MRI data were acquired one day post-injury. Hyperintense lesions on FLAIR (A, C), suggesting traumatic axonal injury (TAI), are seen in the posterior limb of the right internal capsule (solid arrow) and the fornix (dotted arrow). SWI data (B, D) reveal that each lesion is associated with a traumatic microbleed, suggesting hemorrhagic TAI. The lesions in the internal capsule and fornix provided a pathophysiologic basis for the patient's left-sided hemiparesis and memory deficits, respectively, which were present on neurological examination at the time of hospital discharge to a rehabilitation facility. Zoomed views of the T2-FLAIR and SWI data are shown in (C, D). 


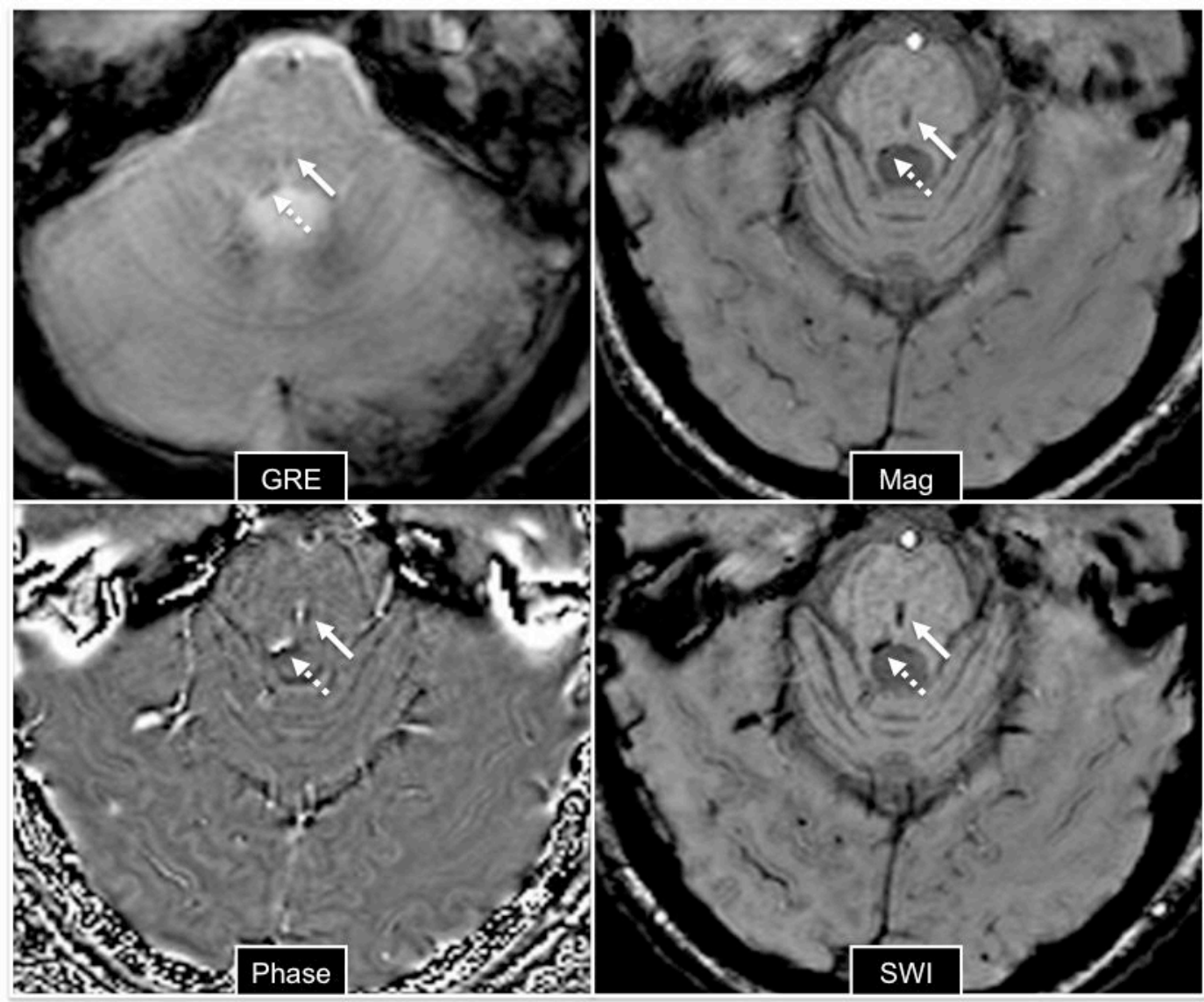

Figure 4.

GRE, Susceptibility Magnitude (Mag), Susceptibility Phase (Phase), and SWI data in a 23year-old woman with severe TBI caused by a motor vehicle accident. The GRE data were acquired on post-TBI day 10 and the SWI, Mag, and Phase data were acquired 5 months post-TBI. A linear focus of hemorrhagic traumatic axonal injury (TAI) located at the midline of the dorsal tegmentum of the pons (solid arrow) is barely perceptible on GRE, but is clearly detectable on SWI. Similarly, a second focus of hemorrhagic TAI in the right dorsolateral quadrant of the pontine tegmentum adjacent to the $4^{\text {th }}$ ventricle (dashed arrow) is difficult to discern on GRE but is clearly demonstrated by SWI. Of note, although the GRE and SWI data were acquired at different times post-injury, the paramagnetic properties of blood are expected to provide similar signal contrast at 10 days and 5 months post-TBI. 

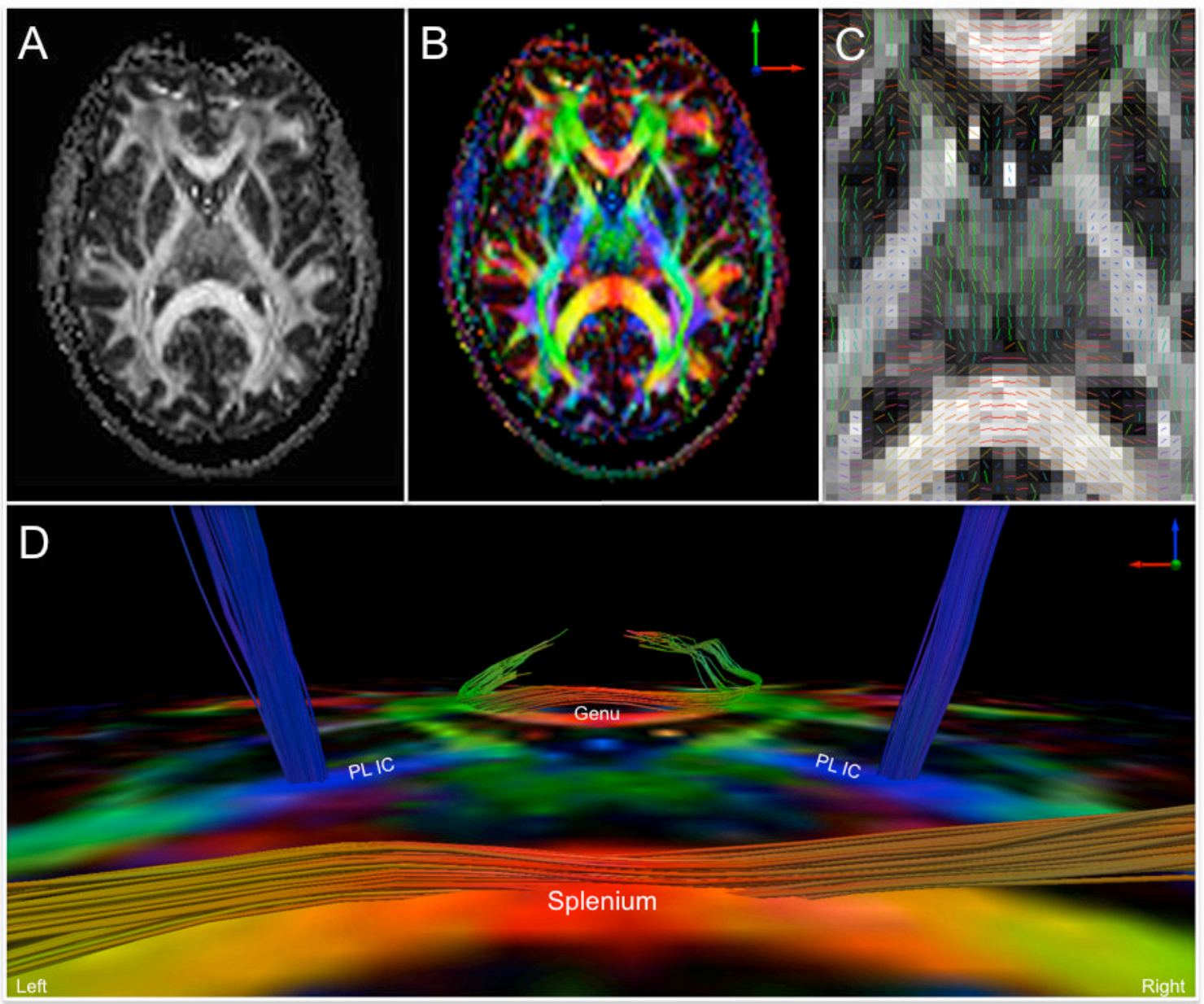

Figure 5.

DTI and diffusion tensor tractography in a 32-year-old healthy man. Greyscale FA map (A), color-coded FA map (B), and color-coded principle eigenvector (i.e. tensor) map (C) are displayed in the axial plane at the level of the mid-thalami. A diffusion tensor tractography analysis is shown in (D) from a posterior perspective, superimposed on the axial color FA map shown in (B). In (A), the signal intensity of the FA map corresponds to the magnitude of the FA in each voxel, on a scale from 0 (black) to 1 (white). In (B, C, and D), colorcoding is according to convention, with medial-lateral fibers coded red, anterior-posterior fibers coded green, and superior-inferior fibers coded blue. In (D), fiber tracts were reconstructed with TrackVis software using a deterministic, streamline tractography model. Tract termination criteria included FA $<0.2$ and intervoxel angle curvature $>60$ degrees. Fiber tracts in the splenium were generated using a single voxel region of interest (ROI) that was manually traced at the center of the splenium. Fiber tracts in the bilateral posterior limbs of the internal capsule (PL IC) were also generated using single voxel ROIs traced at the centers of the PL IC. Tracts in the genu were generated using a single voxel ROI traced in the forceps minor fasciculus of the right frontal lobe. 

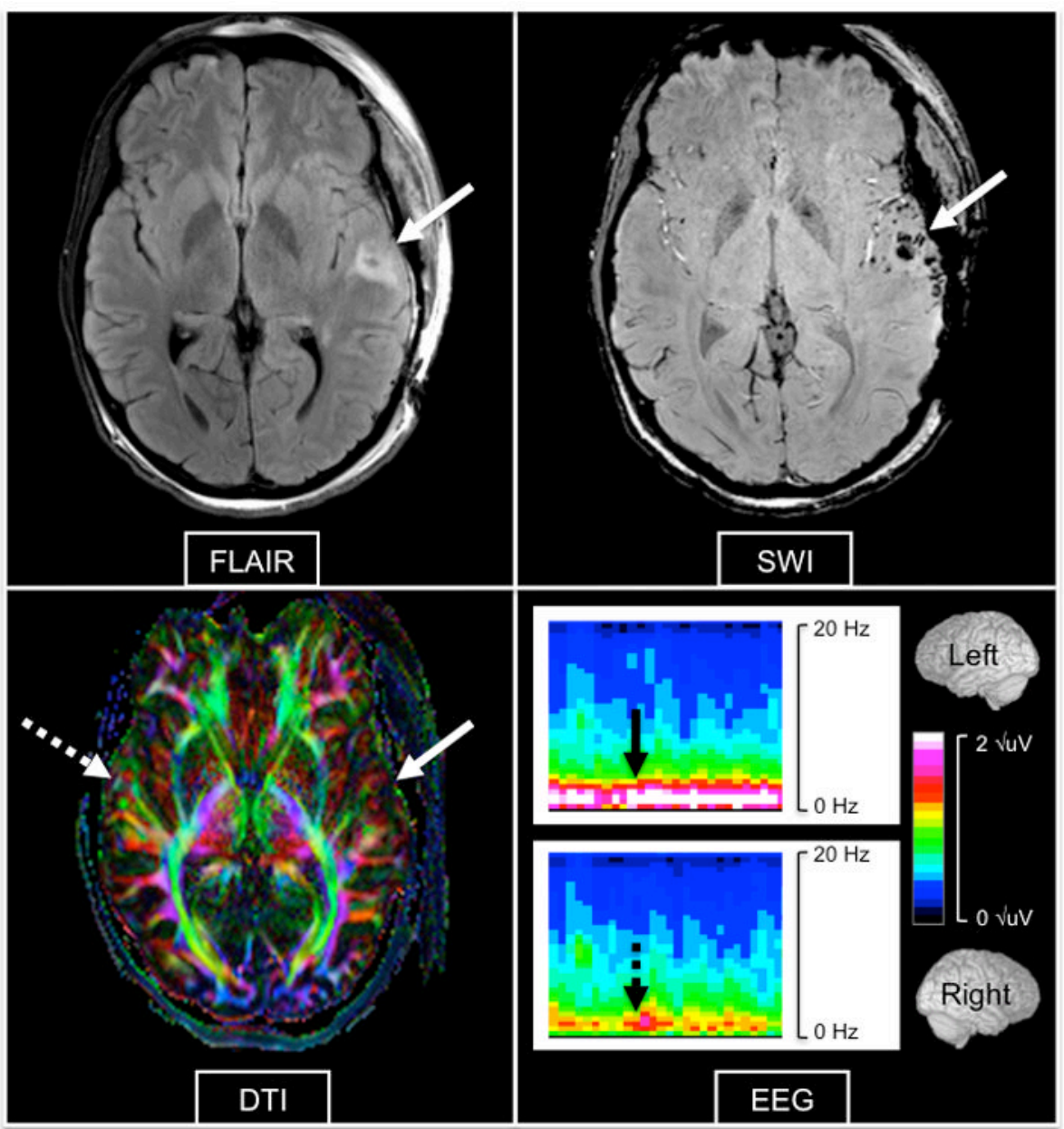

Figure 6.

Multimodal advanced neuroimaging analysis of language deficits in a 20-year-old man with moderate TBI (admission Glasgow Coma Scale score $=9$ ) due to an assault. The patient underwent urgent elevation of a depressed left fronto-temporo-parietal skull fracture prior to MRI data acquisition, which was performed on post-TBI day 2 with a 3 Tesla Siemens Skyra MRI scanner. EEG data were acquired on post-TBI day 1 using a standard 10-20 electrode placement. T2-FLAIR, SWI, and DTI (color FA) data are shown in the axial plane at the level of the superior temporal gyrus (Wernicke's area). EEG power spectrograms are shown for the left (top) and right (bottom) hemispheres, representing EEG data acquired over a 10-minute epoch. In the left superior temporal gyrus (solid arrows), the multimodal imaging data show the following constellation of findings: edema on T2-FLAIR, multiple traumatic microbleeds (TMBs) on SWI (which were not detected by T2-FLAIR); and a relative absence of arcuate fasciculus fibers on the DTI color FA map, as compared to the contralateral superior temporal gyrus (dashed arrow). On the EEG spectrograms, time is represented on the $\mathrm{x}$-axis, frequency is represented on the $\mathrm{y}$-axis, and the color-coding indicates the power of each frequency at each time point (power units $=$ square root of microvolts). In this patient, frequencies in the theta (4 to $8 \mathrm{~Hz}$ ) and alpha ( 8 to $13 \mathrm{~Hz})$ range are present with equal power in the left and right hemispheres. However, delta slowing $(<4$ $\mathrm{Hz}$ ) occurs with much greater power in the left hemisphere, as indicated by the bright white band in the delta frequency range (solid black arrow), as compared to the red-yellow band in the right hemisphere's delta range (dashed black arrow). This left-sided delta slowing on 
EEG corresponds to the left temporal lobe injury revealed by the multimodal imaging data, as well as the mixed expressive and receptive aphasia that were observed on the patient's neurological examination. 


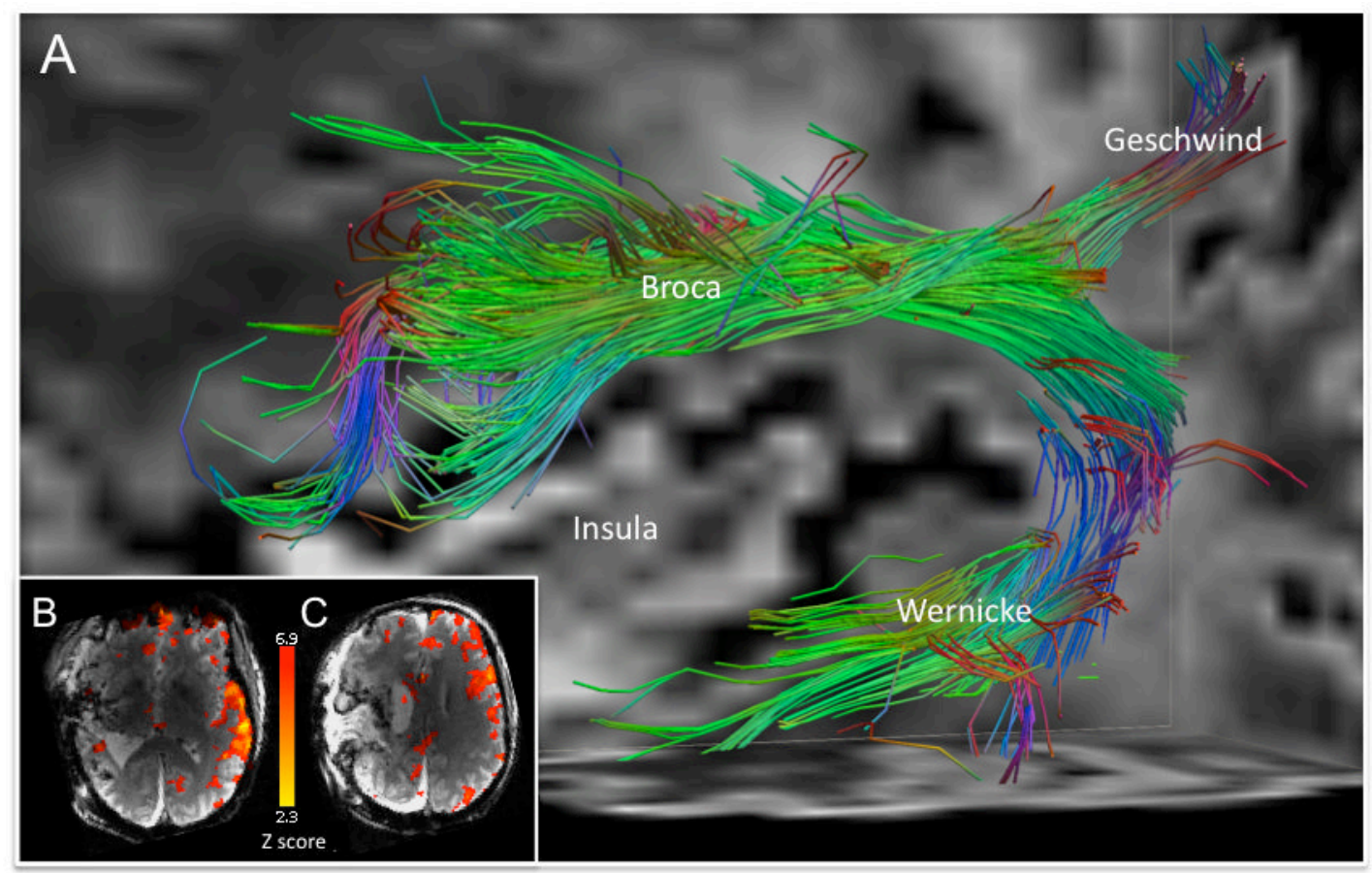

Figure 7.

HARDI tractography and fMRI of the left-sided peri-Sylvian language network in a 27-yearold man with severe TBI caused by a motorcycle accident. Despite extensive contusions, traumatic axonal injury (TAI), skull fractures, subdural and epidural hemorrhages causing acute traumatic coma and requiring urgent right-sided hemicraniectomy, the patient regained the ability to express and understand language prior to hospital discharge. The HARDI data (A) and fMRI data (B, C) were acquired on post-TBI day 16. HARDI tractography analysis using the left arcuate fasciculus as a seed region of interest demonstrates structural connectivity between Wernicke's area in the superior temporal gyrus and Broca's area in the inferior frontal gyrus. In addition, there is preserved arcuate fasciculus connectivity with Geschwind's area, which is comprised of Brodmann areas 39 and 40 within the inferior parietal lobe, as described by Catani et al. ${ }^{264}$ Consistent with these structural connectivity findings, BOLD fMRI analysis during a passive language paradigm (the subject listened to a spoken narrative while in the MRI scanner) demonstrated activation within Wernicke's area (B) and Broca's area (C). HARDI and fMRI data were acquired on a 3 Tesla Siemens Skyra MRI scanner using a 32-channel head coil. The HARDI sequence utilized 60 directional diffusion gradients applied at a $b$ value of $2000 \mathrm{sec} / \mathrm{mm}^{2}$ and with a voxel size of $2 \times 2 \times 2$ $\mathrm{mm}$. Tractography reconstructions were performed with TrackVis software using a deterministic, streamline algorithm and a tract termination threshold of intervoxel angle curvature $>60$ degrees (no FA threshold). The fMRI analysis was performed using FEAT (FMRI Expert Analysis Tool) Version 5.98, part of FSL (FMRIB's Software Library, www.fmrib.ox.ac.uk/fsl). ${ }^{265} \mathrm{Z}$ (Gaussianised T/F) statistic images were thresholded using clusters determined by $\mathrm{Z}>2.3$ and a (corrected) cluster significance threshold of $\mathrm{P}=0.05$ (see color bar inset, in which the color intensity corresponds to the $\mathrm{Z}$ score). 


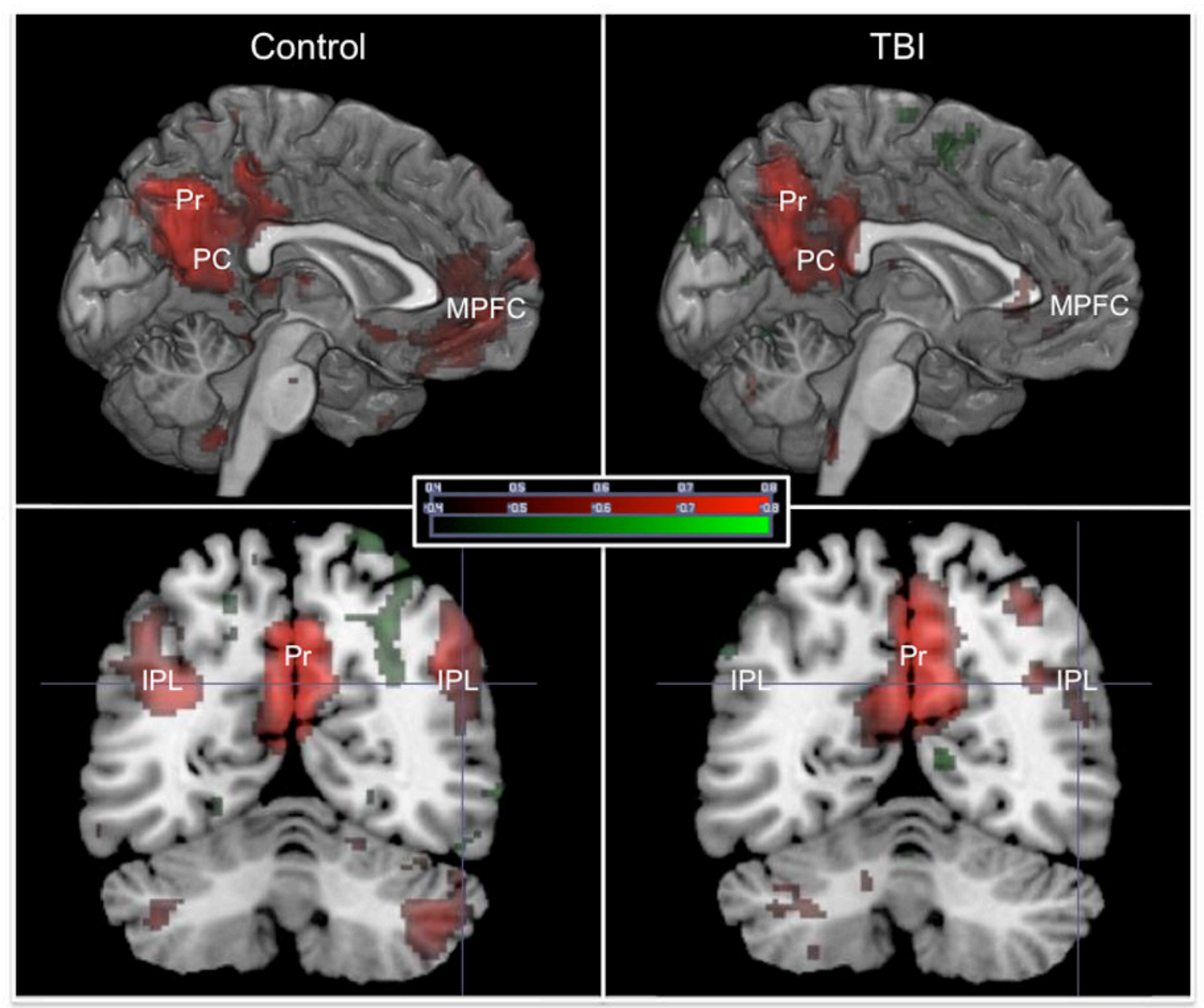

Figure 8.

Resting state fMRI (rs-fMRI) analysis of default mode network (DMN) connectivity in a 17year-old boy with mild TBI due to a sports concussion, as compared to a gender- and agematched control subject. Rs-fMRI analysis of DMN connectivity was performed using a seed region placed in the precuneus (Pr) using techniques previously described. ${ }^{266} \mathrm{~A}$ sagittal view of DMN connectivity in the left hemisphere is shown in the top row using MRIcroGL, with red color indicating positive resting correlations in the BOLD signal and green color indicating negative correlations in the BOLD signal (see inset). In the control subject, functional connectivity is observed between the medial prefrontal cortex (MPFC) and the $\operatorname{Pr}$ and posterior cingulate (PC), whereas in the TBI patient, the MPFC is functionally disconnected from the posterior nodes of the DMN. A coronal view of DMN functional connectivity is shown in the bottom row at the level of the posterior parietal lobe, using the same Pr region of interest. Functional connectivity between the Pr and the bilateral inferior parietal lobules (IPL) is present in the control subject but not in the TBI patient. 